Supporting Information

for

\title{
Iron-Catalyzed Alkylation of Alkenyl Grignard Reagents
}

\author{
Gérard Cahiez,* Christophe Duplais and Alban Moyeux \\ Laboratoire de Synthèse Organique Sélective et de Chimie Organométallique (SOSCO), UMR 8123 CNRS-UCP- \\ ESCOM, 5 mail Gay Lussac, Neuville sur Oise 95031 Cergy-Pontoise Cedex, France \\ gerard.cahiez@u-cergy.fr
}

\section{List of contents}

I/ Influence of the Nature of the Catalytic System

II/ General

III/ Preparation of Alkenyl Halides

IV/ Preparation of Alkenyl Grignard Reagents

1/ General Procedure

2/ Stereospecific Preparation of (Z)-Propenylmagnesium Bromide

IV/ Iron-Catalyzed Cross-Coupling Reaction: General Procedure 


\section{I/ Influence of the Nature of the Catalytic System}

It should be noted that the reaction can also be performed in the presence of 1.6 equivalents of TMEDA ( $\mathrm{RMgBr} / \mathrm{TMEDA}=1: 1)$ according to the procedure recently described by Nakamura ${ }^{1}$ for the iron-catalyzed aryl-alkyl coupling. (Table S1, entry 2). However, this procedure is less efficient since the yield is clearly lower and the amount of amine is higher (Entries 1 and 2).

Table S1

\begin{tabular}{|c|c|c|c|}
\hline Entry & $\begin{array}{c}\text { RMgX } \\
\text { Number of equiv. } \\
\end{array}$ & Conditions $^{\mathrm{a}}$ & $\begin{array}{c}\text { Yield } \\
(\%)\end{array}$ \\
\hline 1 & 1.5 & $5 \%\left[\mathrm{Fe}(\mathrm{acac})_{3} / \mathrm{TMEDA} / \mathrm{HMTA}\right](1: 2: 1)$ & 75 \\
\hline 2 & 1.6 & $5 \% \mathrm{FeCl}_{3}, 160 \% \mathrm{TMEDA}^{\mathrm{b}}$ & 50 \\
\hline
\end{tabular}

${ }^{\mathrm{a}}$ All reactions were performed on a $5 \mathrm{mmol}$ scale in $\mathrm{THF}$ at $0^{\circ} \mathrm{C} .{ }^{\mathrm{b}} \mathrm{RMgBr} / \mathrm{TMEDA}=1: 1$, the amine has to be mixed with the Grignard prior to the addition.

\section{II/ General}

All reactions were carried out under a nitrogen atmosphere. Starting materials were purchased from commercial sources and used without any further purification. Anhydrous THF was purchased from Carlo Erba. All products were purified by distillation. Purity of isolated compounds ranges from 99 to $96 \%$ as determined by GC analysis (capillary column HP-5MS; $30 \mathrm{~m} \times 0.25 \mathrm{~mm} \times 0.25 \mu \mathrm{m})$. All compounds give satisfactory centesimal analysis. The analytical data for the known compounds were found to match with the literature data.

${ }^{1} \mathrm{H}$ and ${ }^{13} \mathrm{C}-\mathrm{NMR}$ spectra were recorded on a JEOL ECX-400 spectrometer. Mass spectra were obtained on a Hewlett-Packard HP 5973 spectrometer via a GC/MS coupling with a HewlettPackard HP 6890 chromatograph equipped wia th a capillary column HP-5MS. Ionisation was obtained by electronic impact (EI, $70 \mathrm{eV}$ ). Mass spectra are reported as $\mathrm{m} / \mathrm{z}$.

The stereochemical purity of the products was determined by gas chromatography analysis. In the case of alkenylmagnesium bromides, the stereochemical purity was determined by iodolysis then $\mathrm{GC}$ analysis of the resulting mixture of $(Z)$ - and $(E)$-alkenyl iodides.

The Grignard solutions were titrated according to the procedure described by Watson ${ }^{2}$. 


\section{III/ Preparation of Alkenyl Halides}

\section{1/ Synthesis of 1-Bromo-2-methylpropene}

This compound is synthesized according to the procedure described by Farrell and Bachman. ${ }^{3}$

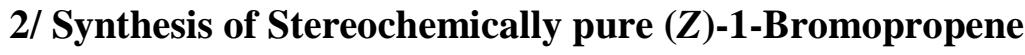

This compound is synthesized according to the procedure described by Fuller and Walker. ${ }^{4}$

\section{IV/ Preparation of Alkenylmagnesium Bromide from Alkenyl Bromide and Magnesium.}

\section{1/ General Procedure}

The alkenyl Grignard reagents used for performing the experiments described in Table 1 were prepared from commercial mixtures of $(Z)$ - and $(E)$-alkenylbromides according to the procedure reported by $\mathrm{H}$. Normant ${ }^{5}$. The proportion of $(Z)$ - and $(E)$-isomers and the temperature employed to prepare the Grignard are given in Table $\mathrm{S} 2$.

\section{Table S2}

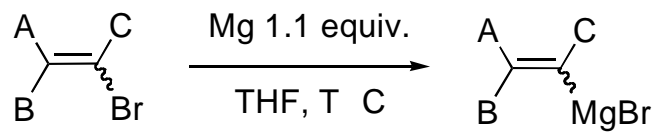

\begin{tabular}{ccccccc}
\hline \multirow{2}{*}{ Entry } & A & B & C & $\begin{array}{c}\text { Alkenyl bromide } \\
\text { E/Z Ratio }\end{array}$ & $\begin{array}{c}\text { Alkenylmagnesium bromide } \\
\text { E/Z Ratio }\end{array}$ & T ${ }^{\circ} \mathbf{C}$ \\
\hline $\mathbf{1}$ & $\mathrm{Me}$ & $\mathrm{H}$ & $\mathrm{H}$ & $80 / 20$ & $86 / 14$ & $10-15^{\circ} \mathrm{C}$ \\
$\mathbf{2}$ & $\mathrm{Me}$ & $\mathrm{H}$ & $\mathrm{Me}$ & $83 / 17$ & $87 / 13$ & $50{ }^{\circ} \mathrm{C}$ \\
$\mathbf{3}$ & $\mathrm{Ph}$ & $\mathrm{H}$ & $\mathrm{H}$ & $88 / 12$ & $78 / 22$ & $0{ }^{\circ} \mathrm{C}$ \\
\hline
\end{tabular}

It is well known that the reaction of magnesium with alkenyl halides takes place with a partial isomerization of the double bond. ${ }^{6}$ However, it is possible to limit the isomerization by working, as far as possible, at a low temperature.

\section{2/ Stereospecific Preparation of ( $Z$ )-Propenylmagnesium Bromide}

Under stirring, 2 to 3 drops of 1,2-dibromoethane were added to magnesium (1.1 equiv., 66 mmol, $1.60 \mathrm{~g})$ in THF $(5 \mathrm{~mL})$. Then, a solution of $(Z)-1$-bromopropene $(Z>99 \%, 1 \mathrm{M}$ solution in 
THF, $60 \mathrm{mmol}, 60 \mathrm{~mL}$ ) was introduced dropwise at $7{ }^{\circ} \mathrm{C}$. Stirring was continued for $4 \mathrm{~h}$ at room temperature. (Z)-1-Propenylmagnesium bromide was obtained in 70\% yield $(\mathrm{Z} / \mathrm{E}=97: 3)$.

\section{IV/ Iron-Catalyzed Cross-Coupling Reaction: General Procedure.}

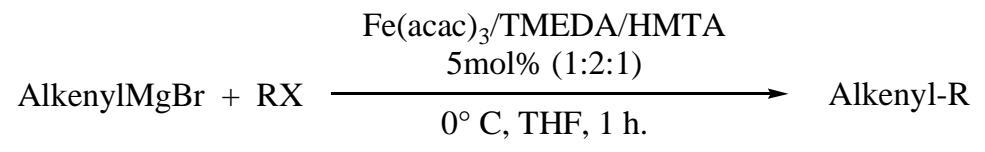

A dry $250 \mathrm{~mL}$ flask, equipped with a mechanical stirrer and a septum, was charged with THF (15 $\mathrm{mL}$ ), alkyl halide (25 mmol), Fe(acac) 3 (443 mg, $1.25 \mathrm{mmol}, 5 \mathrm{~mol} \%$ ), TMEDA (290 mg, 2.5 mmol, $10 \mathrm{~mol} \%$ ) and $\mathrm{HMTA}^{\mathrm{a}}$ (175 $\left.\mathrm{mg}, 1.25 \mathrm{mmol}, 5 \mathrm{~mol} \%\right)$. The reaction mixture was cooled to $0^{\circ} \mathrm{C}$ then the alkenylmagnesium bromide $(37.5 \mathrm{mmol})$, as a solution in THF, was added dropwise for $1 \mathrm{~h}$. After completion of the addition, the reaction mixture was stirred for an additional $30 \mathrm{~min}$, then quenched with a $1 \mathrm{M}$ aqueous $\mathrm{HCl}$ solution $(100 \mathrm{~mL})$. The aqueous phase was extracted with petroleum ether $(3 \times 30 \mathrm{~mL})$, then the combined organic layers were dried with $\mathrm{MgSO}_{4}$ and concentrated in vacuo or distilled under atmospheric pressure, according to the boiling point of the product. The crude residue was purified by distillation at a reduced pressure to afford the coupling product as a colorless oil (55-84\%).

${ }^{a} H M T A=$ hexamethylmethylentetramine 


\section{V/ Spectral Data}

\section{3-Methyl-1-nonene (entry 1)}

Colorless oil. b.p. $=43{ }^{\circ} \mathrm{C} / 10$ Torr

${ }^{1} \mathrm{H}-\mathrm{NMR}\left(400 \mathrm{MHz}, \mathrm{CDCl}_{3}\right) \delta 0.77(\mathrm{t}, \mathrm{J}=6.87 \mathrm{~Hz}, 3 \mathrm{H}), 0.82(\mathrm{~d}, \mathrm{~J}=6.87 \mathrm{~Hz}, 3 \mathrm{H}), 1.17(\mathrm{~m}$, $10 \mathrm{H}), 2.01(\mathrm{~m}, 1 \mathrm{H}), 4.85(\mathrm{~m}, 2 \mathrm{H}), 5.62(\mathrm{~m}, 1 \mathrm{H})$.

${ }^{13} \mathrm{C}-\mathrm{NMR}\left(100 \mathrm{MHz}, \mathrm{CDCl}_{3}\right) \delta 14.13(\mathrm{CH} 3), 17.93(\mathrm{CH} 3), 22.70(\mathrm{CH} 2), 28.95(\mathrm{CH} 2), 29.22$ (CH2), 29.36 (CH2), 29.55 (CH2), 29.65 (CH2), 31.93 (CH2), 32.62 (CH2), $124.51(\mathrm{CH}), 131.70$ $\mathrm{CH})$.

Microanalysis : Calcd. $\mathrm{C}=85.63 \mathrm{H}=14.37$; Found : $\mathrm{C}=85.39 \mathrm{H}=14.61$

$\operatorname{MS}(\mathrm{EI}, 70 \mathrm{eV}) \mathrm{m} / z[\mathrm{M}+\mathrm{H}]^{+}=126$

\section{4-Methyl-2-decene (entry 2) E/Z : 85/15}

Colorless oil. b.p. $=59^{\circ} \mathrm{C} / 10$ Torr

${ }^{1} \mathrm{H}-\mathrm{NMR}\left(400 \mathrm{MHz}, \mathrm{CDCl}_{3}\right) \delta 0.77(\mathrm{t}, \mathrm{J}=6.87 \mathrm{~Hz}, 3 \mathrm{H}), 0.82(\mathrm{~d}, \mathrm{~J}=6.87 \mathrm{~Hz}, 3 \mathrm{H}), 1.17(\mathrm{~m}$, $10 \mathrm{H}), 1.55(\mathrm{dd}, \mathrm{J}=6.87 \mathrm{~Hz}$ and $\mathrm{J}=1.83 \mathrm{~Hz}, 0.15 \times 3 \mathrm{H}), 1.57(\mathrm{dd}, \mathrm{J}=6.87 \mathrm{~Hz}$ and $\mathrm{J}=1.83 \mathrm{~Hz}$, $0.85 \times 3 \mathrm{H}), 1.95(\mathrm{~m}, 0.85 \times 1 \mathrm{H}), 2.35(\mathrm{~m}, 0.15 \times 1 \mathrm{H}), 5.2(\mathrm{~m}, 2 \mathrm{H})$.

${ }^{13} \mathrm{C}-\mathrm{NMR}\left(100 \mathrm{MHz}, \mathrm{CDCl}_{3}\right) \delta 12.96$ (0.15 x CH3), 14.21 (0.85 x CH3), $18.04(\mathrm{CH} 3), 20.91$ (CH3), 22.78 (CH2), 27.41 (CH2), 29.57 (CH2), 31.21 (0.15 x CH), 32.01 (0.85 x CH), 36.79 (CH2), 37.31 (0.85 x CH2), 37.55 (0.15 x CH2), 122.00 (0.15 x CH), 122.67 (0.85 x CH), 137.43 (0.15 x CH), $137.85(0.85 \times \mathrm{CH})$.

Microanalysis: Calcd. $\mathrm{C}=85.63 \mathrm{H}=14.37$; Found : $\mathrm{C}=85.96 \mathrm{H}=14.04$

$\operatorname{MS}(\mathrm{EI}, 70 \mathrm{eV}) \mathrm{m} / z[\mathrm{M}+\mathrm{H}]^{+}=154$

\section{3,4-Dimethyl-2-decene (entry 3) E/Z : 85/15}

Colorless oil. b.p. $=88^{\circ} \mathrm{C} / 10$ Torr

${ }^{1} \mathrm{H}-\mathrm{NMR}\left(400 \mathrm{MHz}, \mathrm{CDCl}_{3}\right) \delta 0.80(\mathrm{t}, \mathrm{J}=7.33 \mathrm{~Hz}, 3 \mathrm{H}), 0.85(\mathrm{t}, \mathrm{J}=6.87 \mathrm{~Hz}, 3 \mathrm{H}), 1.18(\mathrm{~m}$, 10H), $1.47(\mathrm{~m}, 6 \mathrm{H}), 1.98(\mathrm{~m}, 0.15 \times 1 \mathrm{H}), 2.55$ (sextuplet, $\mathrm{J}=7.79 \mathrm{~Hz}, 0.85 \mathrm{x} 1 \mathrm{H}), 5.11$ (q, J = $5.95 \mathrm{~Hz}, 1 \mathrm{H})$.

${ }^{13} \mathrm{C}-\mathrm{NMR}\left(100 \mathrm{MHz}, \mathrm{CDCl}_{3}\right) \delta 12.79(\mathrm{CH} 3), 14.11(\mathrm{CH} 3), 17.94(\mathrm{CH} 3), 19.07$ (CH3), 22.69 (CH2), 27.67 (CH2), $29.55(\mathrm{CH} 2), 31.91(\mathrm{CH} 2), 33.29(\mathrm{CH} 2), 34.83(\mathrm{CH}), 118.53(\mathrm{CH}), 140.04$ $(\mathrm{Cq})$.

Microanalysis: Calcd. $\mathrm{C}=85.63 \mathrm{H}=14.37$; Found $: \mathrm{C}=85.28 \mathrm{H}=14.72$

MS (EI, $70 \mathrm{eV}) m / z[\mathrm{M}+\mathrm{H}]^{+}=168$ 


\section{2,4-Dimethyl-2-decene (entry 4)}

Colorless oil. b.p. $=92{ }^{\circ} \mathrm{C} / 10$ Torr

${ }^{1} \mathrm{H}-\mathrm{NMR}\left(400 \mathrm{MHz}, \mathrm{CDCl}_{3}\right) \delta 0.80(\mathrm{t}, \mathrm{J}=6.87 \mathrm{~Hz}, 3 \mathrm{H}), 0.80(\mathrm{t}, \mathrm{J}=6.87 \mathrm{~Hz}, 3 \mathrm{H}), 1.16(\mathrm{~m}, 10$ $\mathrm{H}), 1.52(\mathrm{~s}, 3 \mathrm{H}), 1.59(\mathrm{~d}, \mathrm{~J}=1.37 \mathrm{~Hz}, 3 \mathrm{H}), 2.2(\mathrm{~m}, 1 \mathrm{H}), 4.78(\mathrm{dt}, \mathrm{J}=9,16 \mathrm{~Hz}$ and $\mathrm{J}=1.37 \mathrm{~Hz}$, $1 \mathrm{H})$.

${ }^{13} \mathrm{C}-\mathrm{NMR}\left(100 \mathrm{MHz}, \mathrm{CDCl}_{3}\right) \delta 14.11(\mathrm{CH} 3), 17.90(\mathrm{CH} 3), 21.29(\mathrm{CH} 3), 22.69(\mathrm{CH} 2), 25.77$ (CH3), 27.48 (CH2), $29.54(\mathrm{CH} 2), 31.95(\mathrm{CH} 2), 32.39(\mathrm{CH}), 37.88(\mathrm{CH} 2), 129.49(\mathrm{CH}), 131.69$ $(\mathrm{CH})$.

Microanalysis: Calcd. $\mathrm{C}=85.63 \mathrm{H}=14.37$; Found : $\mathrm{C}=85.52 \mathrm{H}=14.48$

MS (EI, $70 \mathrm{eV}) \mathrm{m} / z[\mathrm{M}+\mathrm{H}]^{+}=168$

\section{3-Cyclohexyl-2-methyl-2-propene ${ }^{8}$ (entry 5)}

Colorless oil. b.p. $=94{ }^{\circ} \mathrm{C} / 100$ Torr

${ }^{1} \mathrm{H}-\mathrm{NMR}\left(400 \mathrm{MHz}, \mathrm{CDCl}_{3}\right) \delta 1.0(\mathrm{~m}, 2 \mathrm{H}), 1.26(\mathrm{~m}, 2 \mathrm{H}), 1.55-1.68(\mathrm{~m}, 5 \mathrm{H}), 1.59(\mathrm{~d}, \mathrm{~J}=0.92$ $\mathrm{Hz}, 3 \mathrm{H}), 1.65(\mathrm{~d}, \mathrm{~J}=1.37 \mathrm{~Hz}, 3 \mathrm{H}), 2.10(\mathrm{~m}, 1 \mathrm{H}), 4.92(\mathrm{~m}, 1 \mathrm{H})$

${ }^{13} \mathrm{C}-\mathrm{NMR}\left(100 \mathrm{MHz}, \mathrm{CDCl}_{3}\right) \delta 17.81(\mathrm{CH} 2), 18.05(\mathrm{CH} 2), 25.77$ (CH2), $26.00(\mathrm{CH} 2), 26.13$ (CH3), 26.37 (CH2), 33.39 (CH3), $33.63(\mathrm{CH} 2), 37.04(\mathrm{CH} 2), 37.28(\mathrm{CH}), 131.19(\mathrm{CH}), 131.43$ $(\mathrm{Cq})$.

Microanalysis: Calcd. $\mathrm{C}=86.88 \mathrm{H}=13.12$; Found $: \mathrm{C}=86.69 \mathrm{H}=13.31$

MS (EI, $70 \mathrm{eV}) \mathrm{m} / z[\mathrm{M}+\mathrm{H}]^{+}=138$

\section{3-Methyl-1-phenyl-1-pentene' (entry 8) E/Z : 76/24}

Colorless oil. b.p. $=72{ }^{\circ} \mathrm{C} / 10$ Torr

${ }^{1} \mathrm{H}-\mathrm{NMR}\left(400 \mathrm{MHz}, \mathrm{CDCl}_{3}\right) \delta 0.86(\mathrm{t}, \mathrm{J}=7.33 \mathrm{~Hz}, 0.24$ x 3H), $0.92(\mathrm{t}, \mathrm{J}=7.33 \mathrm{~Hz}, 0.76$ x 3H), 1.04 (d, J $=6.87 \mathrm{~Hz}, 0.24 \times 3 \mathrm{H}), 1.09(\mathrm{~d}, \mathrm{~J}=6.41 \mathrm{~Hz}, 0.76 \times 3 \mathrm{H}), 1.35$ (quint., $\mathrm{J}=7.33 \mathrm{~Hz}, 0.24 \times 2 \mathrm{H}$ ), 1.40 (quint., $\mathrm{J}=7.33 \mathrm{~Hz}, 0.76 \times 2 \mathrm{H}), 2.2(\mathrm{~m}, 0.76 \times 1 \mathrm{H}), 2.67(\mathrm{~m}, 0.24 \times 1 \mathrm{H}), 5.44\left(\mathrm{dd},{ }^{3} \mathrm{~J}_{\text {cis }}=11.45 \mathrm{~Hz}\right.$ and $\mathrm{J}=10.53$ $\mathrm{Hz}, 0.24 \mathrm{x} 1 \mathrm{H}), 6.11\left(\mathrm{dd},{ }^{3} \mathrm{~J}_{\text {trans }}=16,03 \mathrm{~Hz}\right.$ and $\left.\mathrm{J}=7.79 \mathrm{~Hz}, 0.76 \mathrm{x} 1 \mathrm{H}\right), 6.35(\mathrm{~m}, 2 \mathrm{H}), 7.28-7.36(\mathrm{~m}, 5 \mathrm{H})$.

${ }^{13} \mathrm{C}-\mathrm{NMR}\left(100 \mathrm{MHz}, \mathrm{CDCl}_{3}\right) \delta 11.83(\mathrm{CH} 3), 20.19$ (0.76 x CH2), 20.65 (0.24 x CH2), 29.78 (CH2), 30.33 (CH3), $33.76(\mathrm{CH}), 38.9(\mathrm{CH}), 125.92$ (4x CHarom), 126.33 (CHarom), 126.71 (2x CHarom), $127.51(\mathrm{CH}), 128.09$ (2x CHarom), $128.41(\mathrm{CH}), 128.60$ (CHarom), $136.74(\mathrm{CH}), 137.95(\mathrm{CH})$.

Microanalysis: Calcd. $\mathrm{C}=89.94 \mathrm{H}=10.06$; Found $: \mathrm{C}=90.12 \mathrm{H}=9.88$

$\operatorname{MS}(\mathrm{EI}, 70 \mathrm{eV}) \mathrm{m} / z[\mathrm{M}+\mathrm{H}]^{+}=160$ 


\section{1-Dodecene (entry 9)}

Colorless oil. b.p. $=80^{\circ} \mathrm{C} / 10$ Torr

${ }^{1} \mathrm{H}-\mathrm{NMR}\left(400 \mathrm{MHz}, \mathrm{CDCl}_{3}\right) \delta 0.87(\mathrm{t}, \mathrm{J}=6.87 \mathrm{~Hz}, 3 \mathrm{H}), 1.34(\mathrm{~m}, 16 \mathrm{H}), 2.02(\mathrm{q}, \mathrm{J}=7.33 \mathrm{~Hz}$, $2 \mathrm{H}), 4.91\left(\mathrm{dd},{ }^{3} \mathrm{~J}_{\text {cis }}=10.07 \mathrm{~Hz}\right.$ and $\left.\mathrm{J}_{\text {gem }}=1.37 \mathrm{~Hz}, 1 \mathrm{H}\right), 4.98(\mathrm{~m}, 1 \mathrm{H}), 5.8(\mathrm{~m}, 1 \mathrm{H})$.

${ }^{13} \mathrm{C}-\mathrm{NMR}\left(100 \mathrm{MHz}, \mathrm{CDCl}_{3}\right) \delta 14.12(\mathrm{CH} 3), 22.69(\mathrm{CH} 2), 28.95(\mathrm{CH} 2), 29.16(\mathrm{CH} 2), 29.35$ (CH2), 29.51 (CH2), 29.62 (2x CH2), $31.91(\mathrm{CH} 2), 33.83(\mathrm{CH} 2), 114.06(\mathrm{CH}), 139.27(\mathrm{CH})$.

Microanalysis: Calcd. $\mathrm{C}=85.63 \mathrm{H}=14.37$; Found : $\mathrm{C}=85.83 \mathrm{H}=14.17$

MS (EI, $70 \mathrm{eV}) \mathrm{m} / z[\mathrm{M}+\mathrm{H}]^{+}=182$

\section{2-Tridecene ${ }^{8}$ (entry 10)}

Colorless oil. b.p. $=96^{\circ} \mathrm{C} / 10$ Torr

${ }^{1} \mathrm{H}-\mathrm{NMR}\left(400 \mathrm{MHz}, \mathrm{CDCl}_{3}\right) \delta 0.87(\mathrm{t}, \mathrm{J}=6.87 \mathrm{~Hz}, 3 \mathrm{H}), 1.28(\mathrm{~m}, 16 \mathrm{H}), 1.60(\mathrm{~d}, \mathrm{~J}=5.95 \mathrm{~Hz}$, $0.15 \times 3 \mathrm{H}), 1.62(\mathrm{~m}, 0.85 \times 3 \mathrm{H}), 2.01(\mathrm{q}, \mathrm{J}=6.87 \mathrm{~Hz}, 2 \mathrm{H}), 5.39(\mathrm{~m}, 2 \mathrm{H})$.

${ }^{13} \mathrm{C}-\mathrm{NMR}\left(100 \mathrm{MHz}, \mathrm{CDCl}_{3}\right) \delta 14.10(\mathrm{CH} 3), 17.90(\mathrm{CH} 3), 22.69(\mathrm{CH} 2), 26.84$ (0.15 x CH2), 29.21 (CH2), 29.36 (2 x CH2), 29.56 (2 x CH2), 29.65 (CH2), 31.93 (CH2), 32.62 (0.85 x CH2), $123.58(0.15 \times \mathrm{CH}), 124.51(0.85 \times \mathrm{CH}), 130.91(0.15 \times \mathrm{CH}), 131.67(0.85 \times \mathrm{CH})$.

Microanalysis: Calcd. $\mathrm{C}=85.63 \mathrm{H}=14.37$; Found $: \mathrm{C}=85.42 \mathrm{H}=14.58$

MS (EI, $70 \mathrm{eV}) \mathrm{m} / z[\mathrm{M}+\mathrm{H}]^{+}=182$

\section{3-Methyl-2-tridecene (entry 11) Z/E : 86/14}

Colorless oil. b.p. $=68^{\circ} \mathrm{C} / 4$ Torr

${ }^{1} \mathrm{H}-\mathrm{NMR}\left(400 \mathrm{MHz}, \mathrm{CDCl}_{3}\right) \delta 0.86(\mathrm{t}, \mathrm{J}=6.41 \mathrm{~Hz}, 3 \mathrm{H}), 1.24(\mathrm{~m}, 16 \mathrm{H}), 1.54(\mathrm{dd}, \mathrm{J}=6.41 \mathrm{~Hz}$ and $\mathrm{J}=1.37 \mathrm{~Hz}, 3 \mathrm{H}), 1.64(\mathrm{t}, \mathrm{J}=1.37 \mathrm{~Hz}, 3 \mathrm{H}), 1.80(\mathrm{t}, \mathrm{J}=7.33 \mathrm{~Hz}, 2 \mathrm{H}), 5.16(\mathrm{~m}, 1 \mathrm{H})$.

${ }^{13} \mathrm{C}-\mathrm{NMR}\left(100 \mathrm{MHz}, \mathrm{CDCl}_{3}\right) \delta 13.21(\mathrm{CH} 3), 14.12(\mathrm{CH} 3), 22.69(\mathrm{CH} 2), 23.38(\mathrm{CH} 3), 27.80$ (CH2), 29.36 (CH2), 29.65 (2 x CH2), 29.70 (2 x CH2), 31.36 (CH2), 31.91 (CH2), 118.61 (0.84 x CH), $136.17(0.16 \times \mathrm{CH}), 136.17(0.16 \times \mathrm{CH}), 136.46(0.84 \times \mathrm{CH})$.

Microanalysis: Calcd. $\mathrm{C}=85.63 \mathrm{H}=14.37$; Found : $\mathrm{C}=85.81 \mathrm{H}=14.19$

MS (EI, $70 \mathrm{eV}) m / z[\mathrm{M}+\mathrm{H}]^{+}=196$ 


\section{2-Methyl-2-undecene (entry 12)}

Colorless oil. b.p. $=87^{\circ} \mathrm{C} / 10$ Torr

${ }^{1} \mathrm{H}-\mathrm{NMR}\left(400 \mathrm{MHz}, \mathrm{CDCl}_{3}\right) \delta 0.86(\mathrm{t}, \mathrm{J}=6.87 \mathrm{~Hz}, 3 \mathrm{H}), 1.25(\mathrm{~m}, 12 \mathrm{H}), 1.58(\mathrm{~s}, 3 \mathrm{H}), 1.67(\mathrm{~d}, \mathrm{~J}=$ $0.92 \mathrm{~Hz}, 3 \mathrm{H}), 1.93$ (q., J = $6.87 \mathrm{~Hz}, 2 \mathrm{H}), 5.10$ (m, 1H).

${ }^{13} \mathrm{C}-\mathrm{NMR}\left(100 \mathrm{MHz}, \mathrm{CDCl}_{3}\right) \delta 14.11(\mathrm{CH} 3), 17.66(\mathrm{CH} 3), 22.69(\mathrm{CH} 2), 25.73(\mathrm{CH} 3), 28.05$ (CH2), 29.35 (2x CH2), 29.57 (CH2), $29.91(\mathrm{CH} 2), 31.91(\mathrm{CH} 2), 124.97(\mathrm{CH}), 131.10(\mathrm{CH})$.

Microanalysis: Calcd. $\mathrm{C}=85.63 \mathrm{H}=14.37$; Found : $\mathrm{C}=85.37 \mathrm{H}=14.63$

MS (EI, $70 \mathrm{eV}) \mathrm{m} / z[\mathrm{M}+\mathrm{H}]^{+}=168$

\section{Ethyl 8-methyl-7-nonenoate (entry 13)}

Colorless oil.

purification : flash chromatography (silica gel), petroleum ether / diethyl ether : 99 / 1.

${ }^{1} \mathrm{H}-\mathrm{NMR}\left(400 \mathrm{MHz}, \mathrm{CDCl}_{3}\right) \delta 1.18(\mathrm{t}, \mathrm{J}=6.87 \mathrm{~Hz}, 3 \mathrm{H}), 1.25(\mathrm{~m}, 4 \mathrm{H}), 1.52$ (s, 3H), 1.54 (quint., $\mathrm{J}=7.33 \mathrm{~Hz}, 2 \mathrm{H}), 1.61(\mathrm{~d}, \mathrm{~J}=0.92 \mathrm{~Hz}, 3 \mathrm{H}), 1.90(\mathrm{~m}, 2 \mathrm{H}), 2.20(\mathrm{t}, \mathrm{J}=7.33 \mathrm{~Hz}, 2 \mathrm{H}), 4.04(\mathrm{q}, \mathrm{J}=$ $6.87 \mathrm{~Hz}, 2 \mathrm{H}), 5.03(\mathrm{~m}, 1 \mathrm{H})$.

${ }^{13} \mathrm{C}-\mathrm{NMR}\left(100 \mathrm{MHz}, \mathrm{CDCl}_{3}\right) \delta 14.20(\mathrm{CH} 3), 17.62(\mathrm{CH} 3), 24.89(\mathrm{CH} 2), 25.67(\mathrm{CH} 3), 27.78$ (CH2), 28.76 (CH2), 29.46 (CH2), $34.33(\mathrm{CH} 2), 60.10$ (CH2), $124.53(\mathrm{CH}), 131.37(\mathrm{Cq}), 173.84$ $(\mathrm{Cq})$.

Microanalysis: Calcd. $\mathrm{C}=72.68 \mathrm{H}=11.18$; Found : $\mathrm{C}=72.51 \mathrm{H}=11.02$

MS (EI, $70 \mathrm{eV}) \mathrm{m} / z[\mathrm{M}+\mathrm{H}]^{+}=198$

\section{9-Methyl-8-decenenitrile (entry 14)}

Colorless oil.

purification : flash chromatography (silica gel), petroleum ether / diethyl ether : 99 / 1.

${ }^{1} \mathrm{H}-\mathrm{NMR}\left(400 \mathrm{MHz}, \mathrm{CDCl}_{3}\right) \delta 1.25(\mathrm{~m}, 4 \mathrm{H}), 1.38(\mathrm{~m}, 2 \mathrm{H}), 1.52$ (d, J = Hz, 3H), 1.58 (quint., J = $7.33 \mathrm{~Hz}, 2 \mathrm{H}), 1.62$ (d, J = 0.92 Hz, 3H), $1.9(\mathrm{~m}, 2 \mathrm{H}), 2.26$ (t, J = $7.33 \mathrm{~Hz}, 2 \mathrm{H}), 5.02(\mathrm{tq}, \mathrm{J}=6.87$ $\mathrm{Hz}$ and $\mathrm{J}=1.37 \mathrm{~Hz}, 1 \mathrm{H})$.

${ }^{13} \mathrm{C}-\mathrm{NMR}\left(100 \mathrm{MHz}, \mathrm{CDCl}_{3}\right) \delta 17.04(\mathrm{CH} 3), 17.59(\mathrm{CH} 2), 25.27(\mathrm{CH} 3), 25.65(\mathrm{CH} 2), 27.73$ (CH2), 28.30 (CH2), 28.51 (CH2), 29.42 (CH2), 119.80 (Cq), $124.34(\mathrm{CH}), 131.47(\mathrm{Cq})$. Microanalysis: Calcd. $\mathrm{C}=79.94 \mathrm{H}=11.59 \mathrm{~N}=8.47$; Found $: \mathrm{C}=80.23 \mathrm{H}=11.68 \mathrm{~N}=8.09$ $\mathrm{MS}(\mathrm{EI}, 70 \mathrm{eV}) \mathrm{m} / \mathrm{z}[\mathrm{M}+\mathrm{H}]^{+}=165$ 


\section{(Z)-4-Methyl-2-decene (Scheme 1)}

Colorless oil. b.p. $=59^{\circ} \mathrm{C} / 10$ Torr

${ }^{1} \mathrm{H}-\mathrm{NMR}\left(400 \mathrm{MHz}, \mathrm{CDCl}_{3}\right) \delta 0.77(\mathrm{t}, \mathrm{J}=6.87 \mathrm{~Hz}, 3 \mathrm{H}), 0.82(\mathrm{~d}, \mathrm{~J}=6.87 \mathrm{~Hz}, 3 \mathrm{H}), 1.17(\mathrm{~m}$, $10 \mathrm{H}), 1.5(\mathrm{dd}, \mathrm{J}=6.87 \mathrm{~Hz}$ and $\mathrm{J}=1.83 \mathrm{~Hz}, 3 \mathrm{H}), 2.35(\mathrm{~m}, 1 \mathrm{H}), 5.03(\mathrm{~m}, 1 \mathrm{H}), 5.2(\mathrm{~m}, 1 \mathrm{H})$.

${ }^{13} \mathrm{C}-\mathrm{NMR}\left(100 \mathrm{MHz}, \mathrm{CDCl}_{3}\right) \delta 12.96(\mathrm{CH} 3), 14.11(\mathrm{CH} 3), 21.13(\mathrm{CH} 3), 22.68(\mathrm{CH} 2), 27.43$ $(\mathrm{CH} 2), 29.49(\mathrm{CH} 2), 31.21(\mathrm{CH} 2), 31.92(\mathrm{CH}), 37.55(\mathrm{CH} 2), 122.00(\mathrm{CH}), 137.43(\mathrm{CH})$.

Microanalysis: Calcd. $\mathrm{C}=85.63 \mathrm{H}=14.37$; Found $: \mathrm{C}=85.96 \mathrm{H}=14.04$

MS (EI, $70 \mathrm{eV}) m / z[\mathrm{M}+\mathrm{H}]^{+}=154$ 


\section{VI/ References}

1. Nakamura, M.; Matsuo, K.; Ito, S.; Nakamura, E. J. Am. Chem. Soc. 2004, 126, 3686.

2. Watson, S. C.; Eastham, J. F. J. Organometal. Chem. 1967, 9, 165.

3. Farrell, J. K. ; Bachman, G. B. J. Am. Chem. Soc. 1935, 57, 1281.

4. Fuller, C. E. ; Walker, D. G. J. Org. Chem. 1991, 56, 4066.

5. a) Normant, H. C. R. Acad. Sci., Ser. C 1954, 239, 1516.

b) Normant H.; Maitte, P. Bull. Soc. Chim. Fr. 1956, 1439

c) Normant, H. Bull. Soc. Chim. Fr. 1957, 728.

6. a) Walborsky, H. M. Acc. Chem. Res. 1990, 23, 286.

b) Yoshino, T.; Manube, Y. J. Am. Chem. Soc. 1963, 85, 2860.

c) Yoshino, T.; Manube, Y.; Kikuchi, Y. J. Am. Chem. Soc. 1964, 86, 4670.

d) Martin, G.J.; Martin M.L. Bull. Soc. Chim. Fr. 1966, 1635.

e) Méchin, B.; Naulet, N. J.Organometal.Chem. 1972, 39, 229.

7. Lin, H.S.; Paquette, L.A. Synthetic Communications. 1994, 24, 2502-2506.

8. Cahiez, G. ; Avedissian, H. Synthesis 1998, 1199.

9. Caubere, P. ; Coudert, G. Tetrahedron 1972, 28, 5635. 


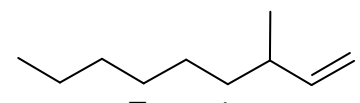

Entry 1

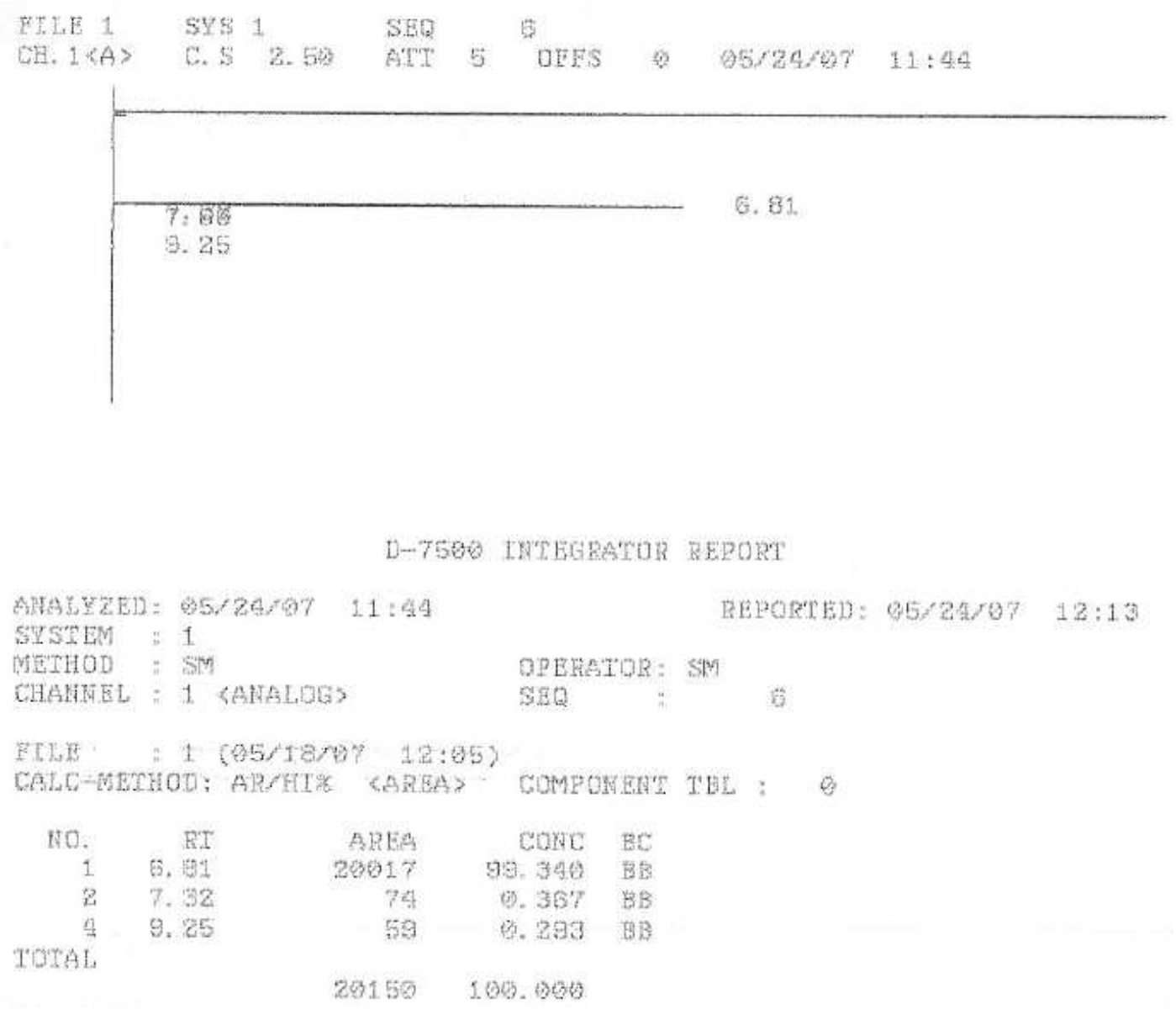




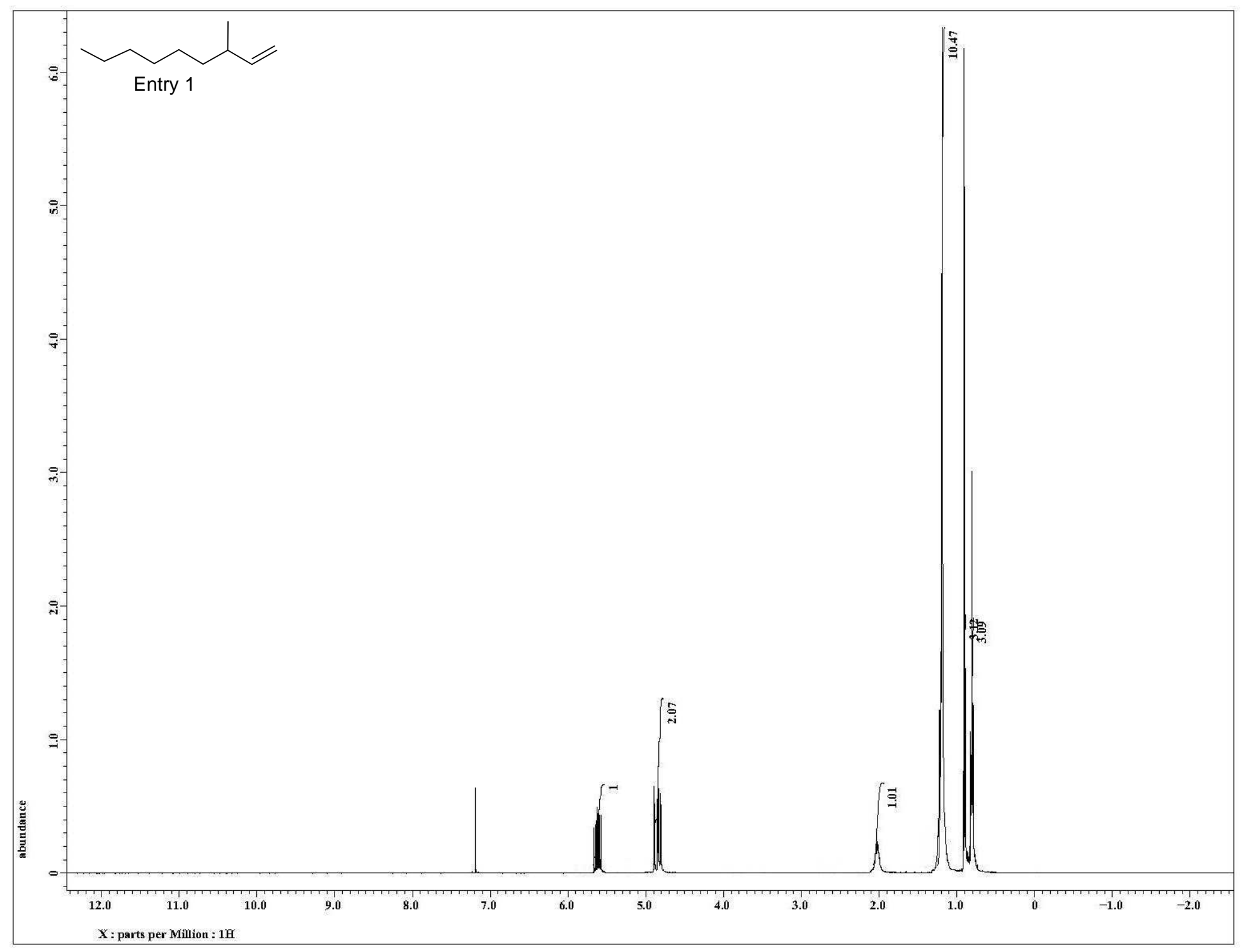

สี 


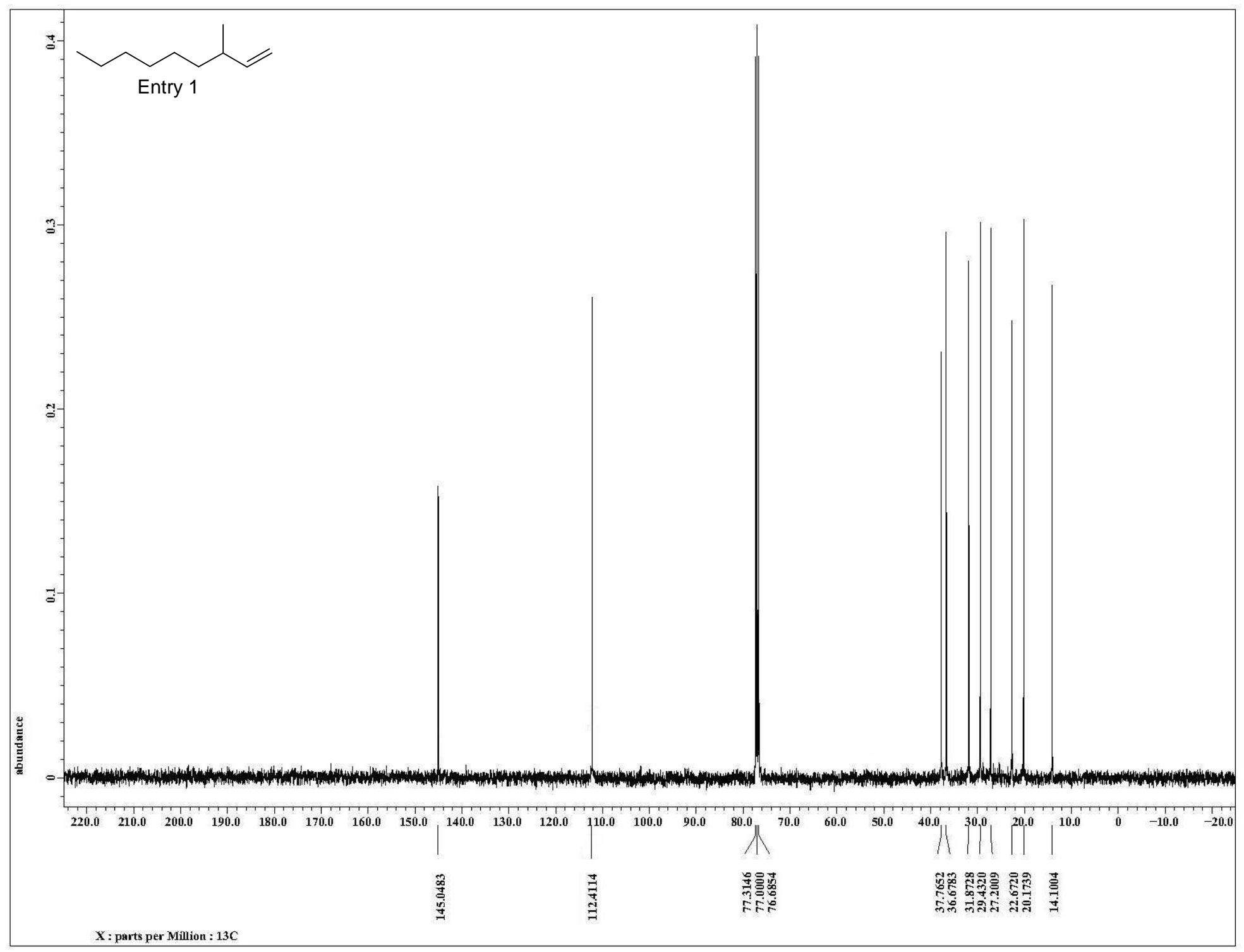




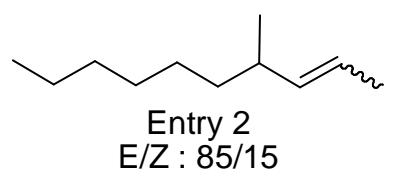

CH. 1 C.S 2.50 ATT O OFFS O BOPQ7/90 D6:25

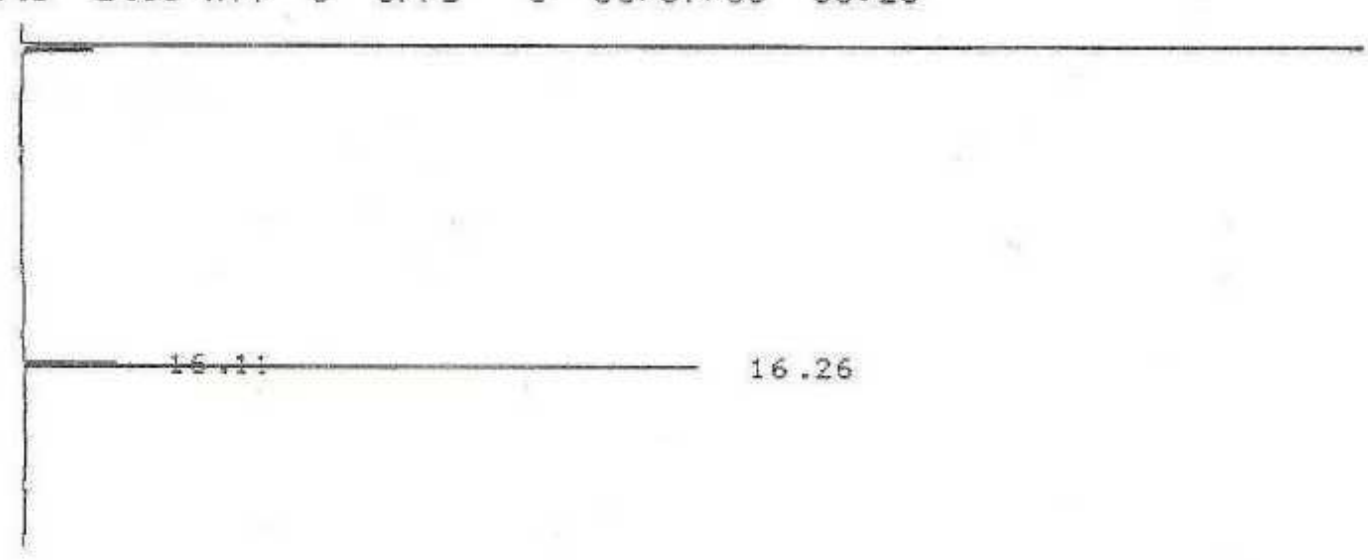

$B-259 B$

$00 \angle 87 \angle 00 \quad 96: 25$

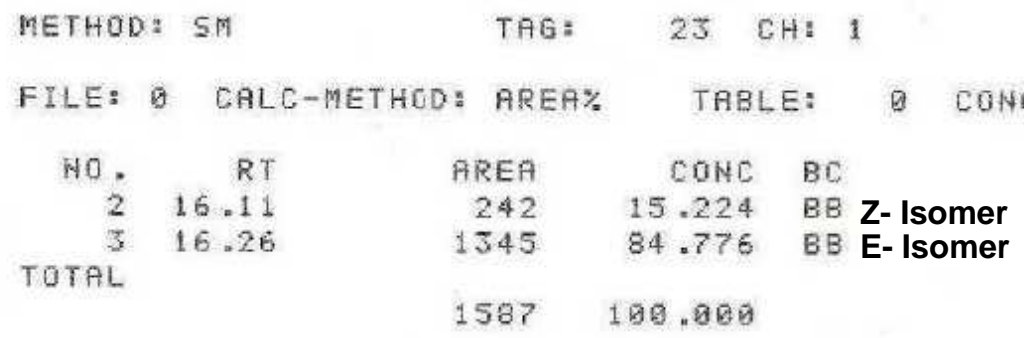




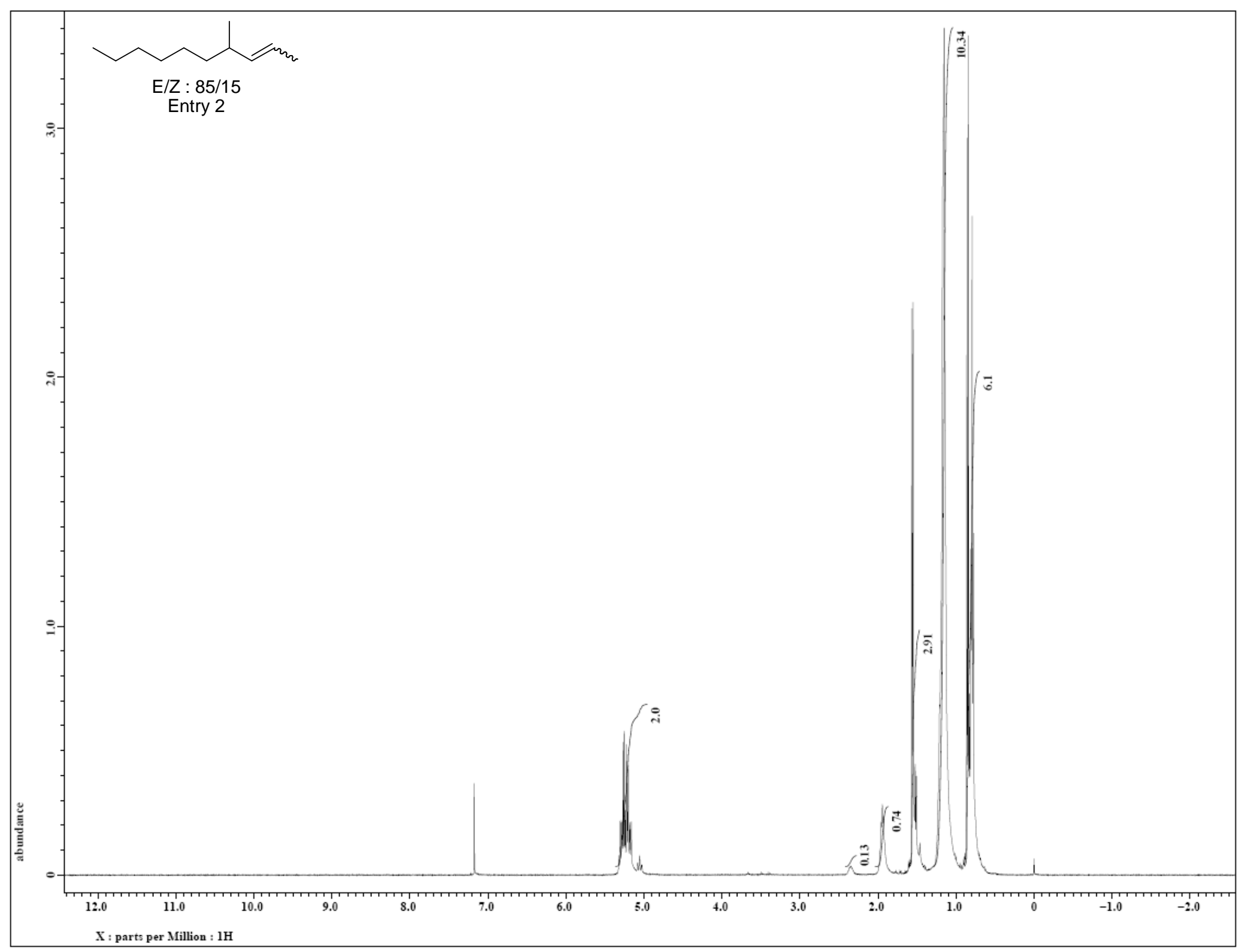

$\frac{10}{2}$ 


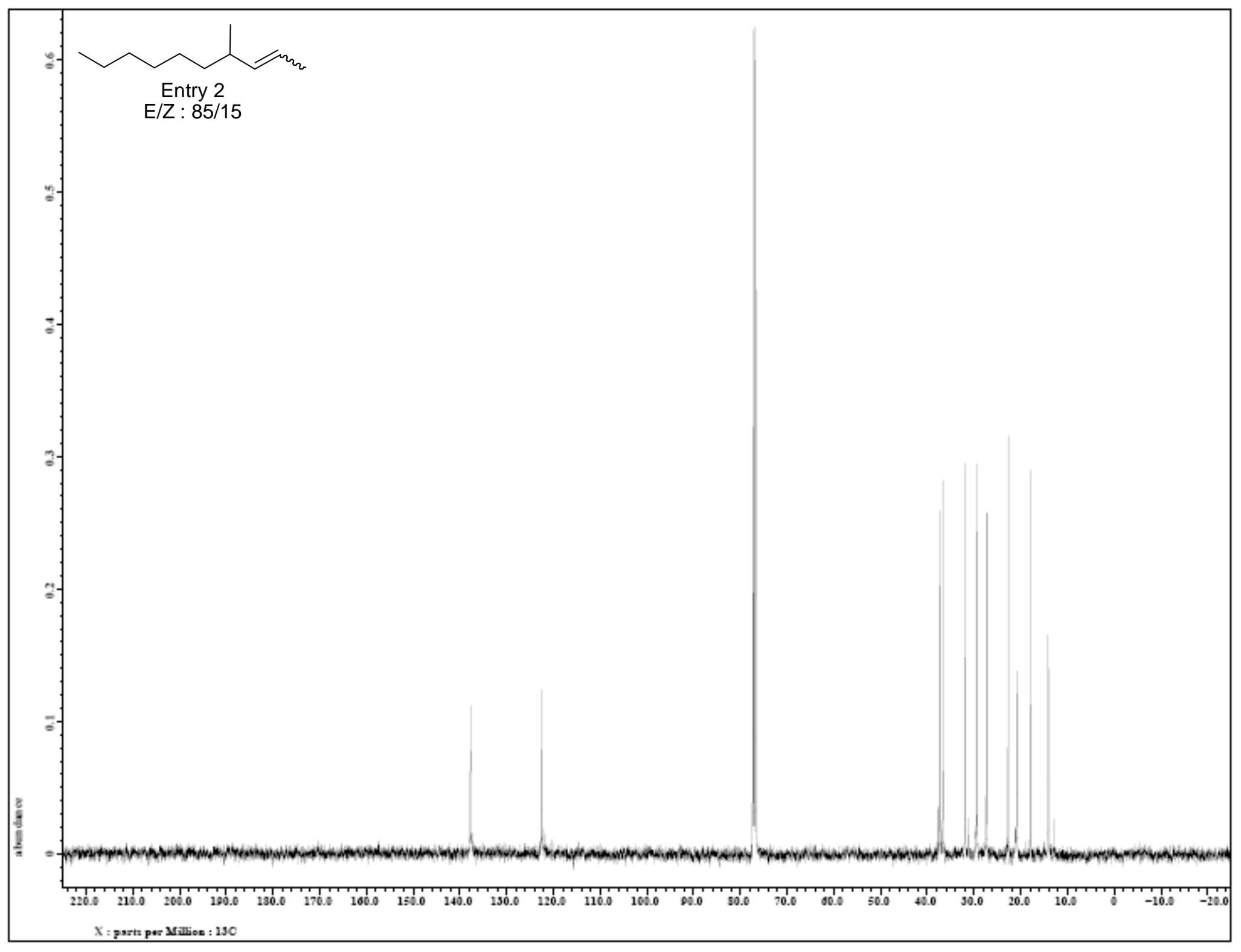




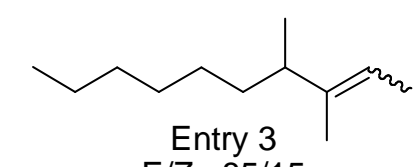

$E / Z: 85 / 15$

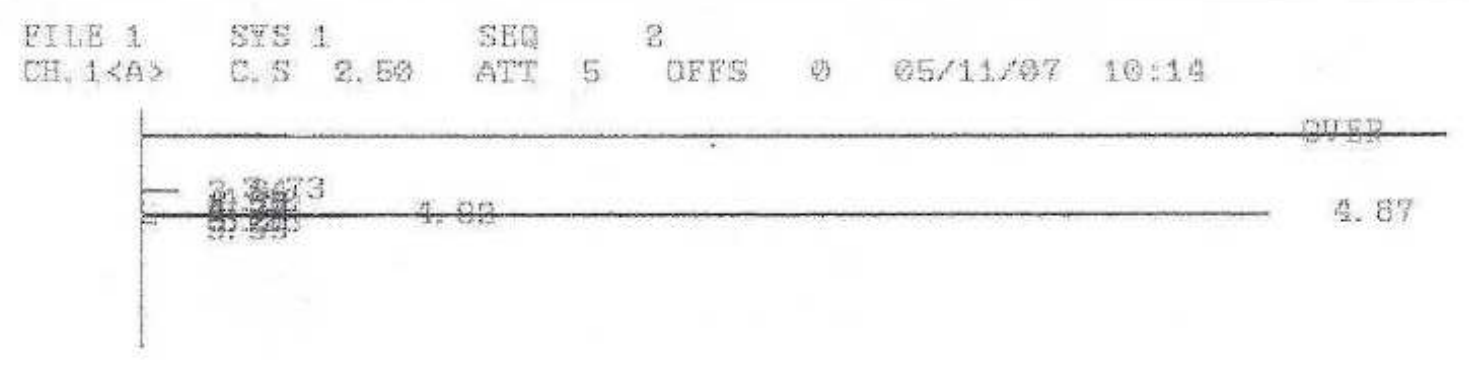

UT-750\% INTEGRATOR REPORT

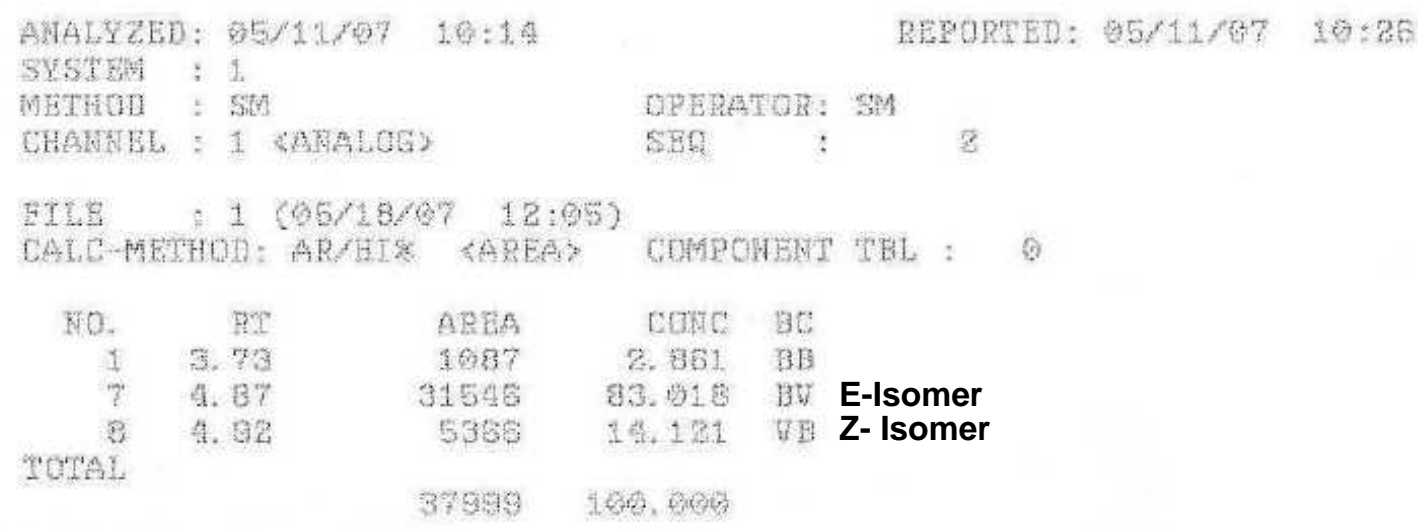




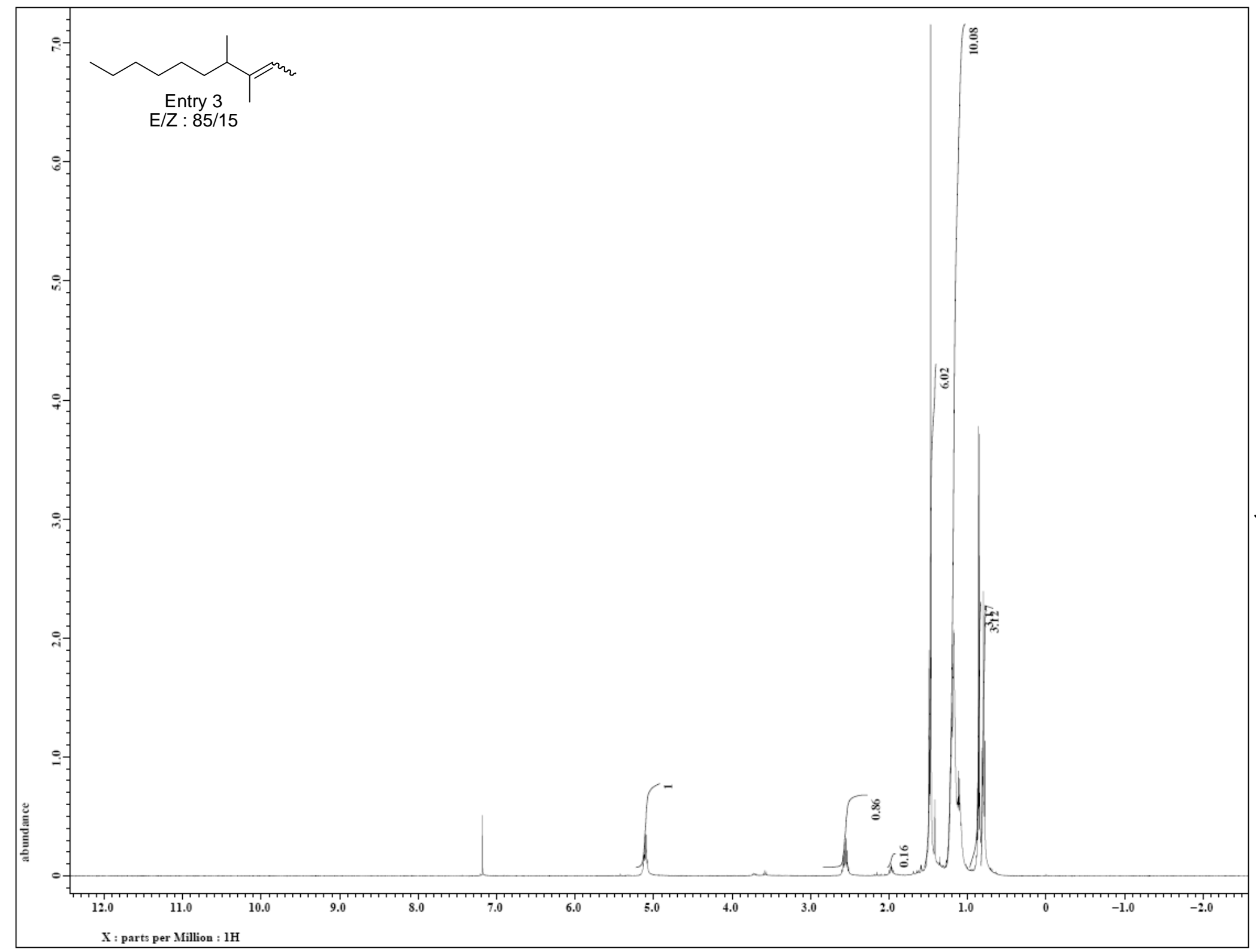




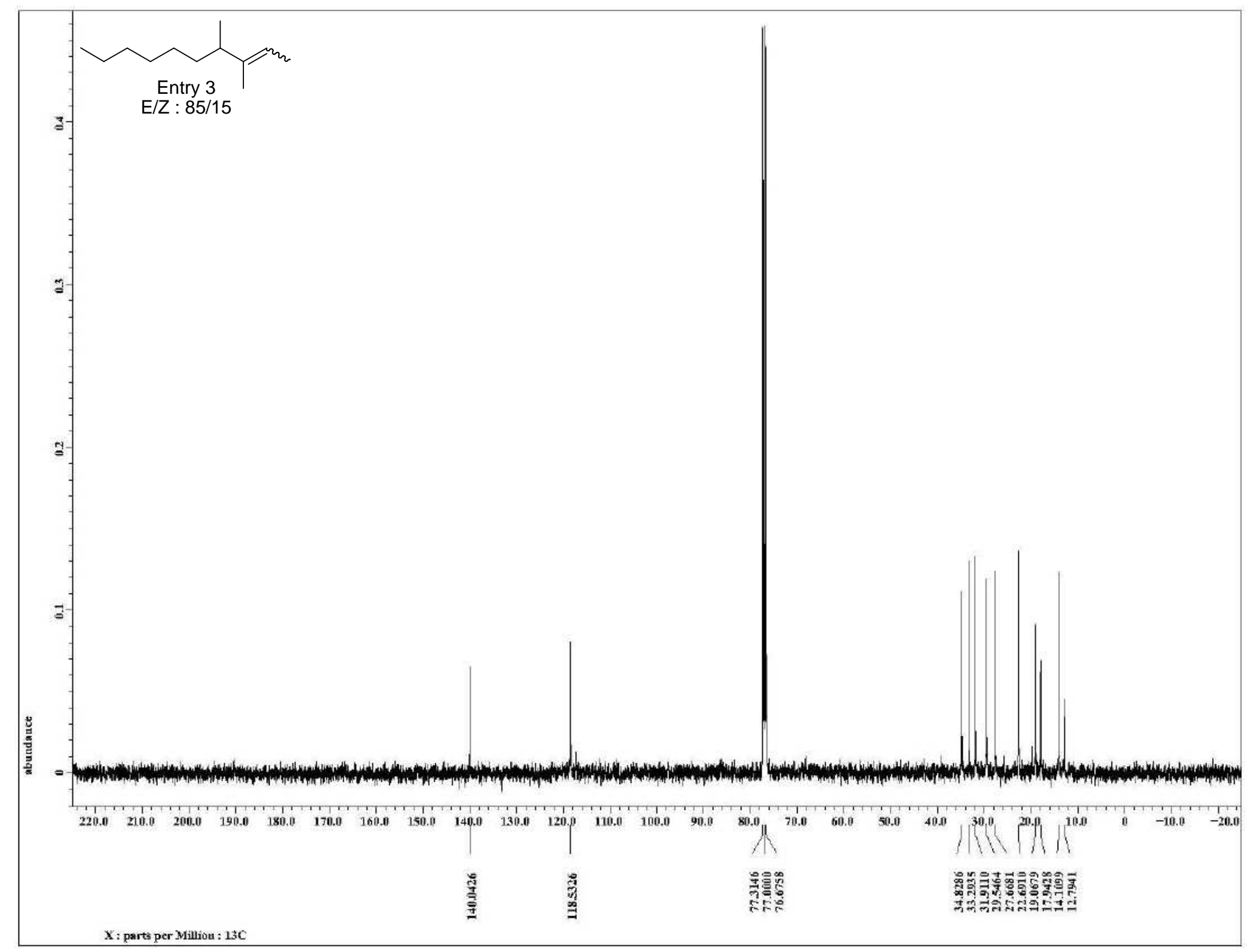

के 


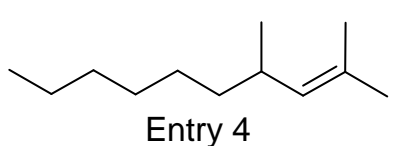

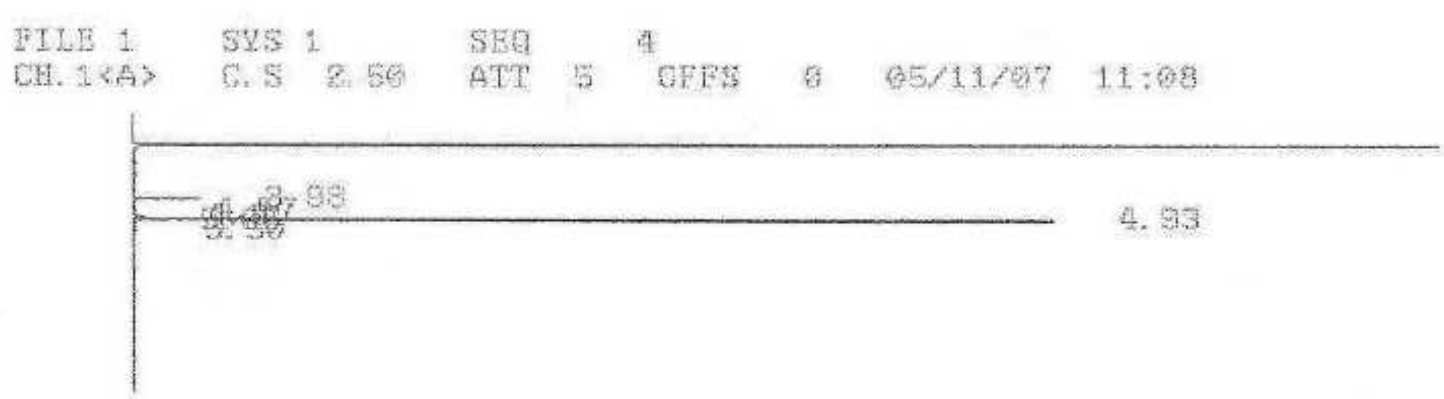

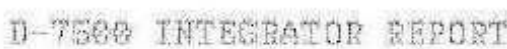

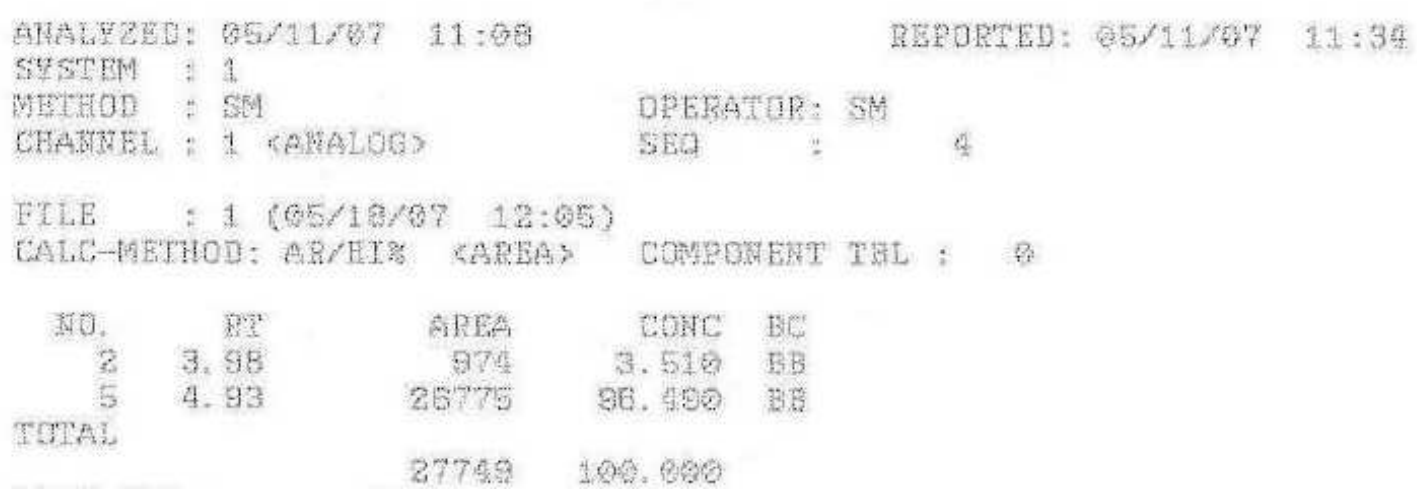




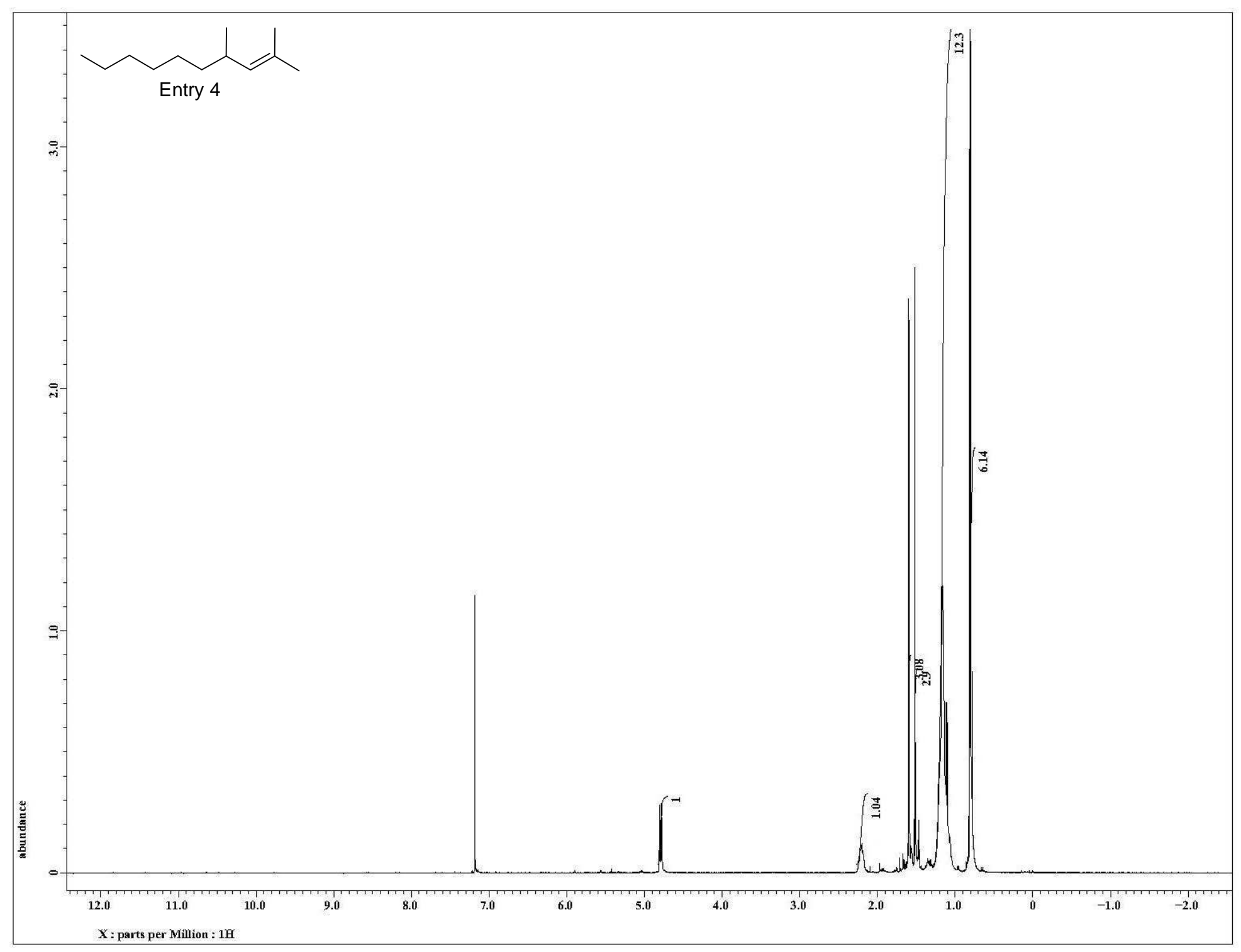




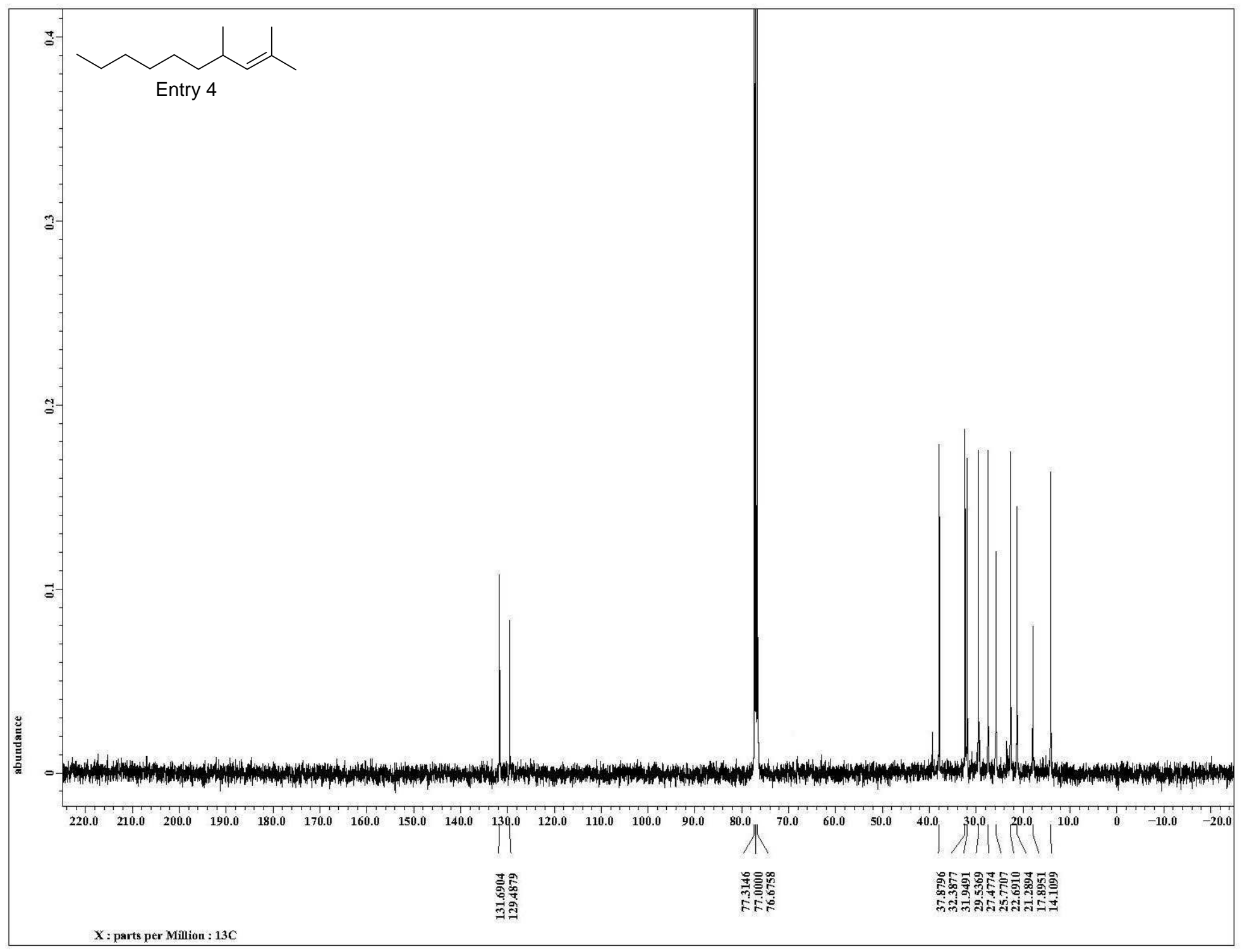




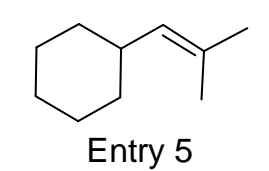

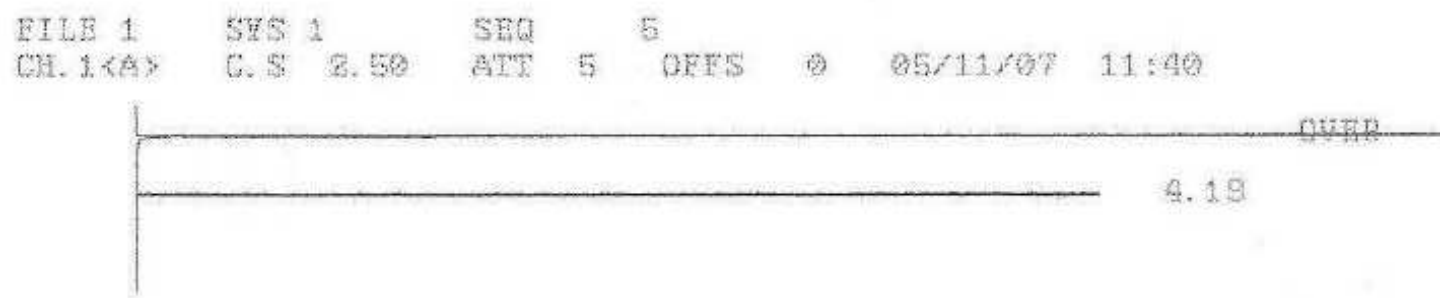

D-7500 THTHPRATOP PEPORT

AHALYZED: Q5511/97 11:46

REDORTED: D5r11,67 11:48

SYSTEM : 1

METHED : S:

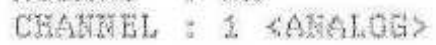

DPERATOH: SA4

(SEQ :

FHE : 1 (O5,1B/OF 12.65$)$

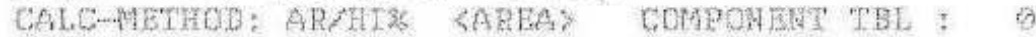

\begin{tabular}{|c|c|c|c|}
\hline 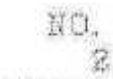 & 4. $\begin{array}{l}79 \\
4.9\end{array}$ & $\begin{array}{r}\text { AREA } \\
27200\end{array}$ & $\begin{array}{r}\text { Cont } \\
106.000\end{array}$ \\
\hline & & 27260 & 106. פण0 \\
\hline
\end{tabular}




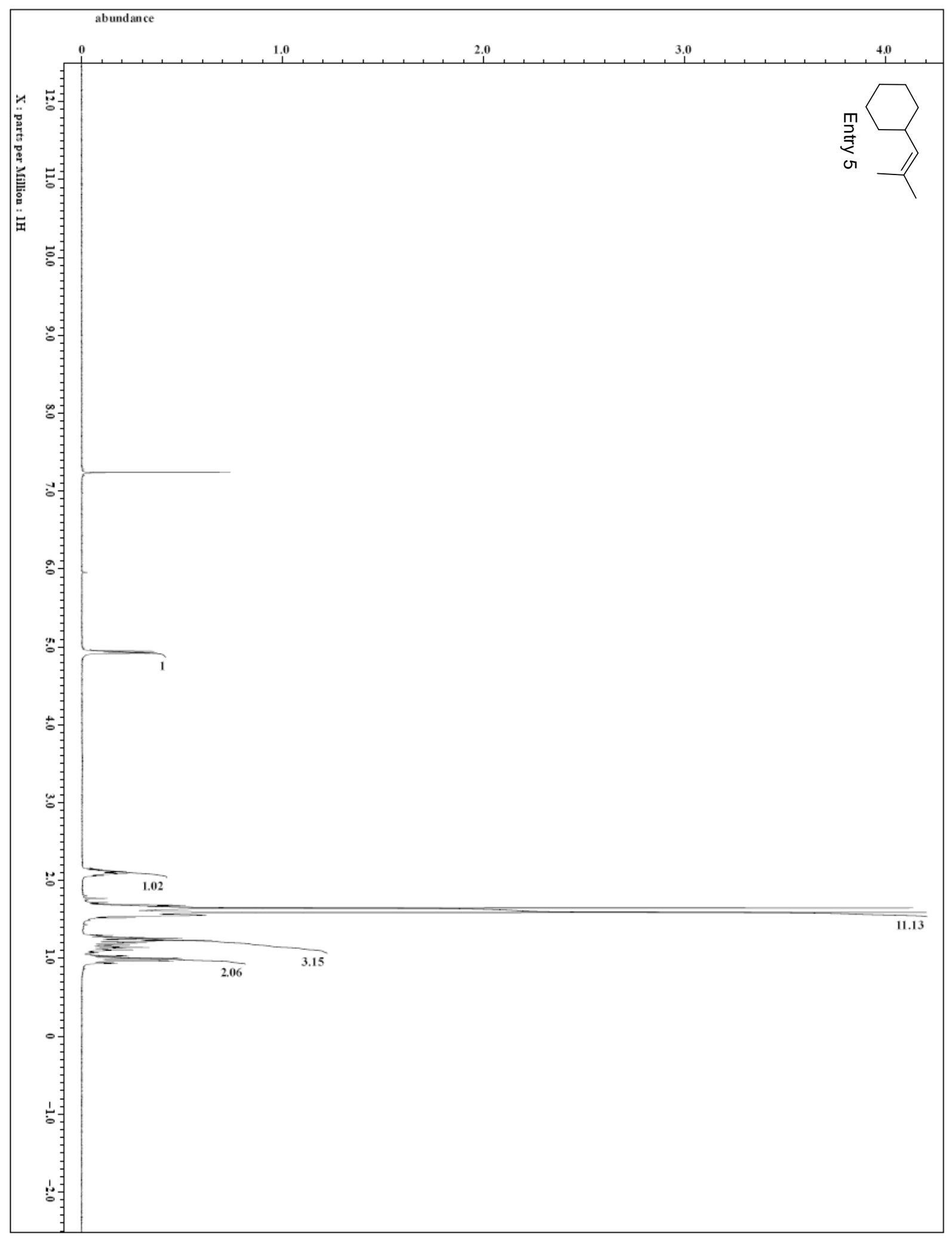

S24 


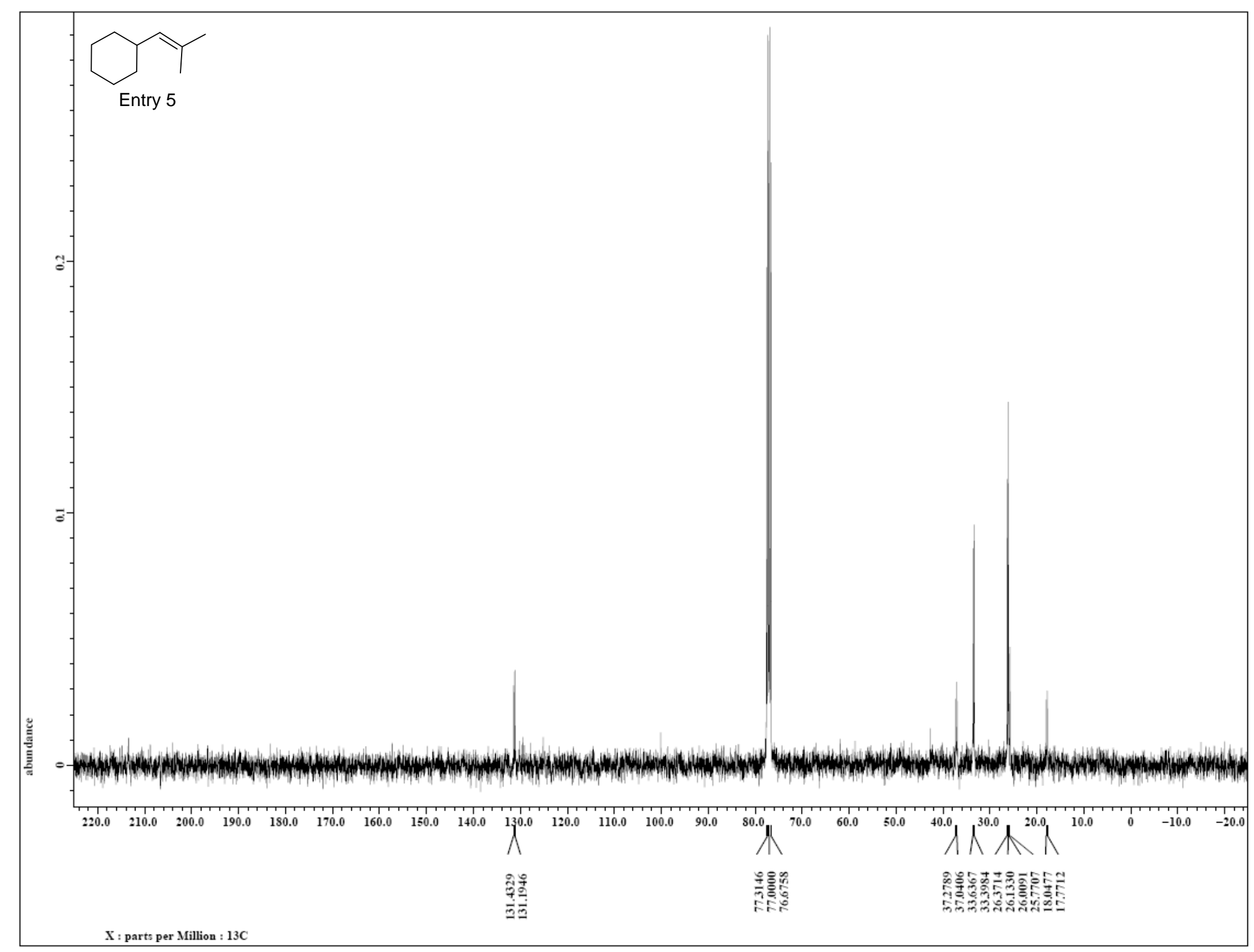




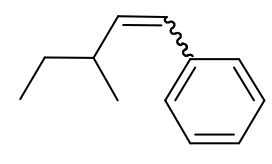

Entry 8

$E / Z: 76 / 24$

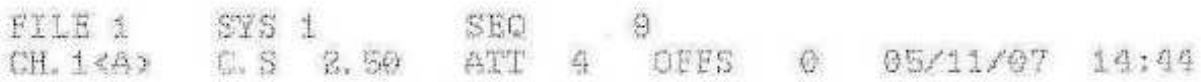

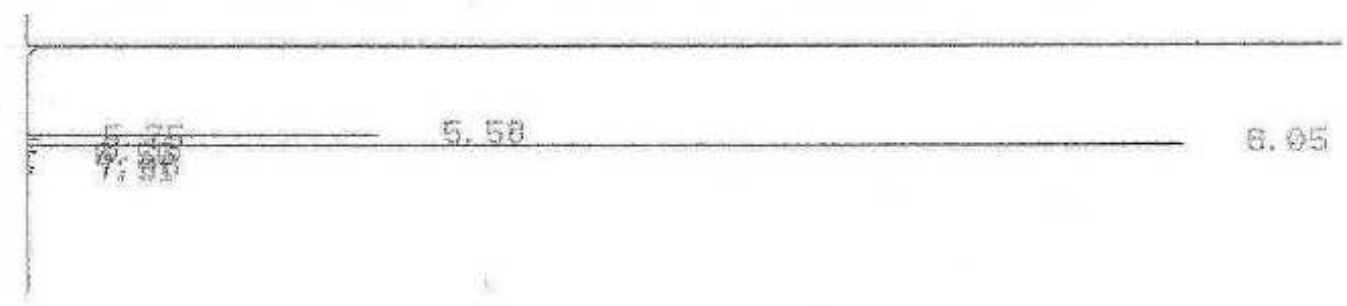

12-75Q6 TNTEGRPBOR FETCRT

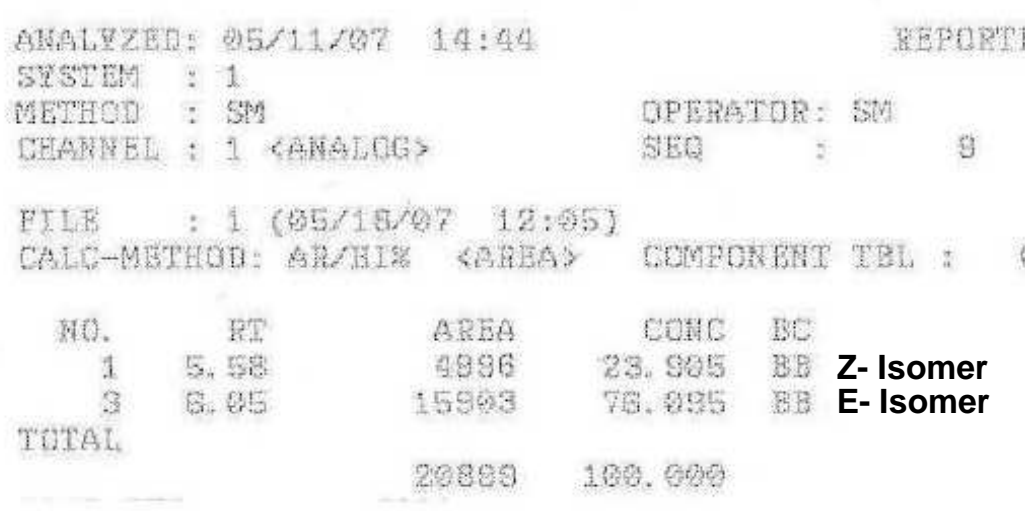




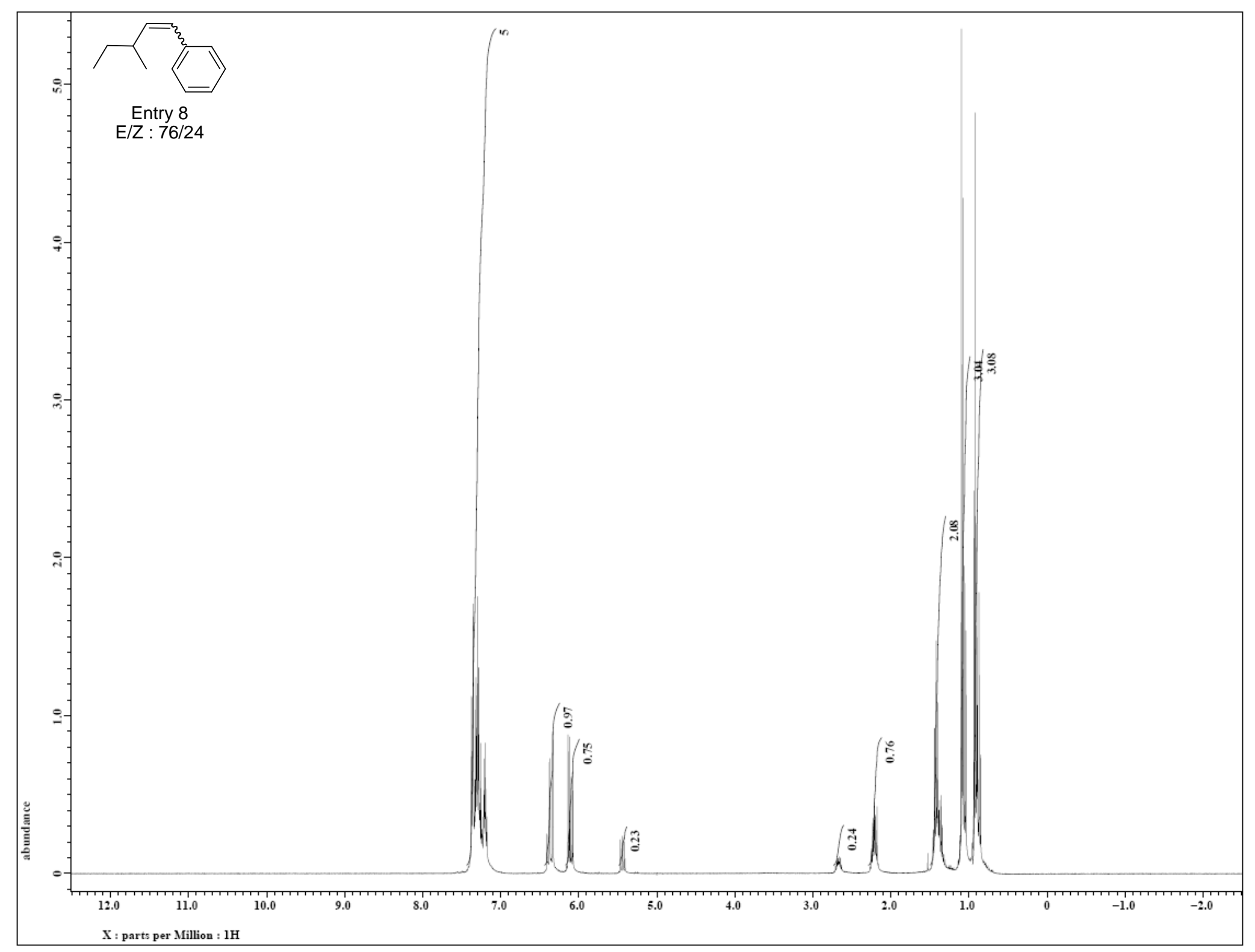

ㅎํ 


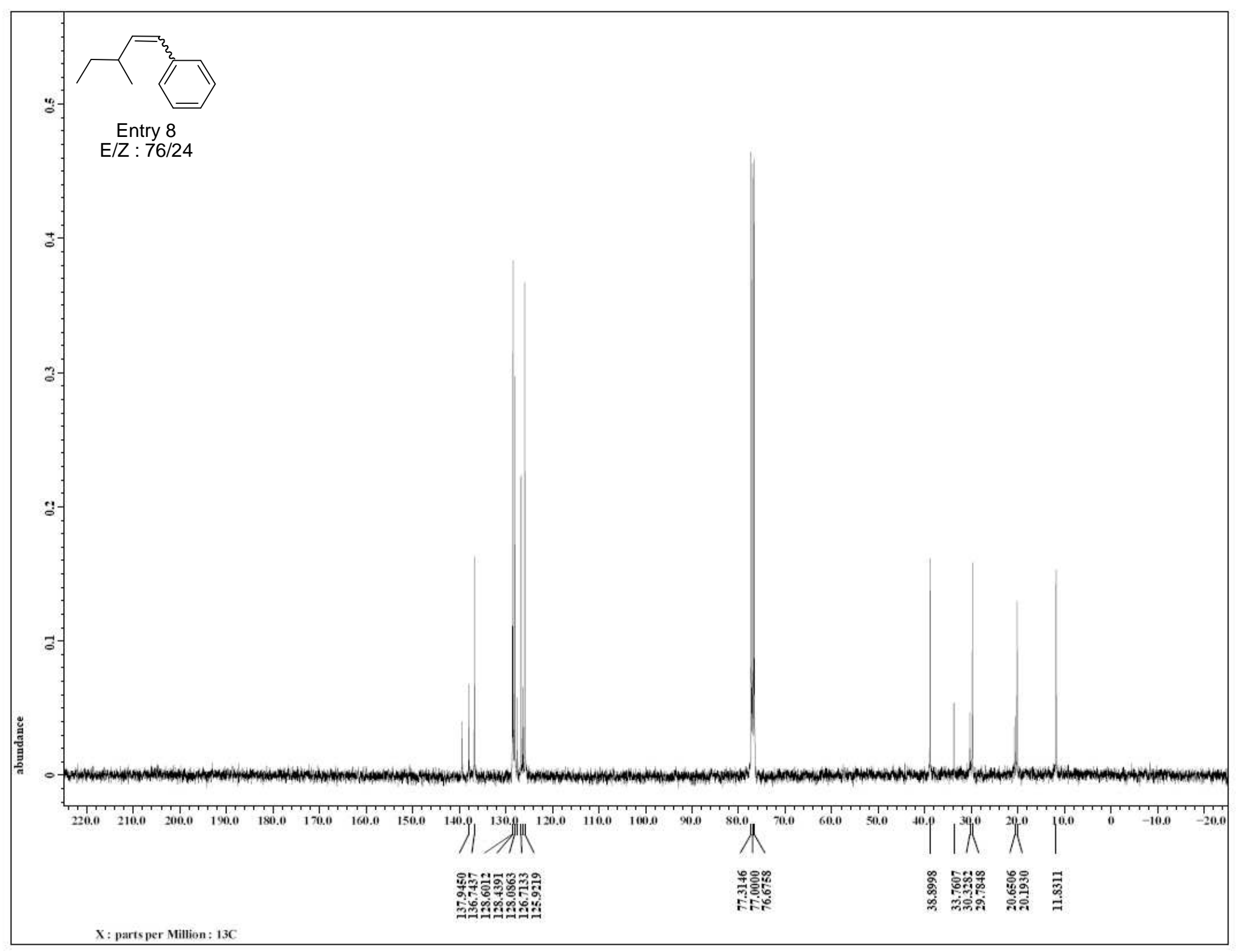




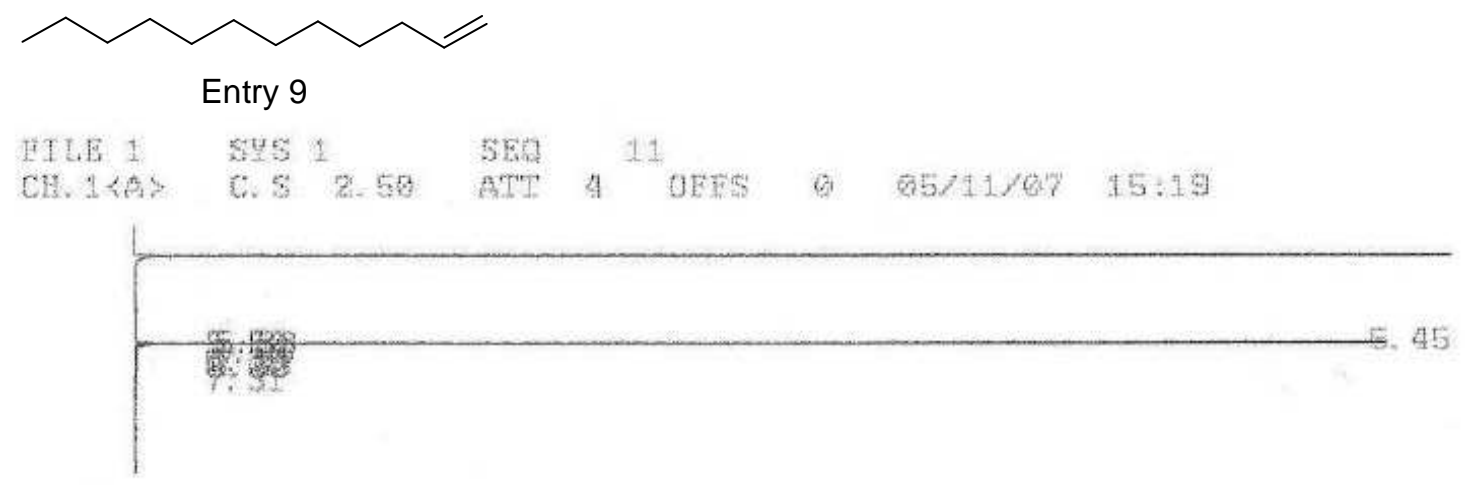

0-7590 IHTHERTOR NEPOR

AMALY2ED: 00711/07 15:19

SEPORTEL: 85,11707 15:33

STST

CHANALL : 1 SANALAG'

OEFRTOR: St

SEO $\quad 11$

ETLE : $1 .(05 / 18 / 27$ 12:05)

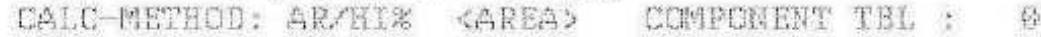

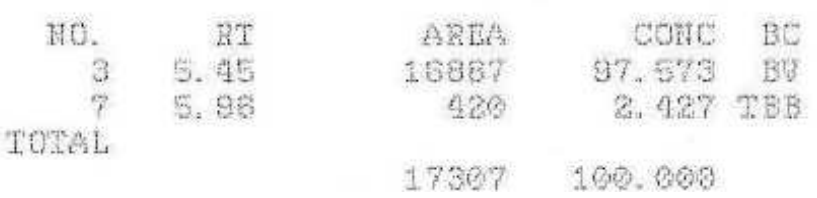




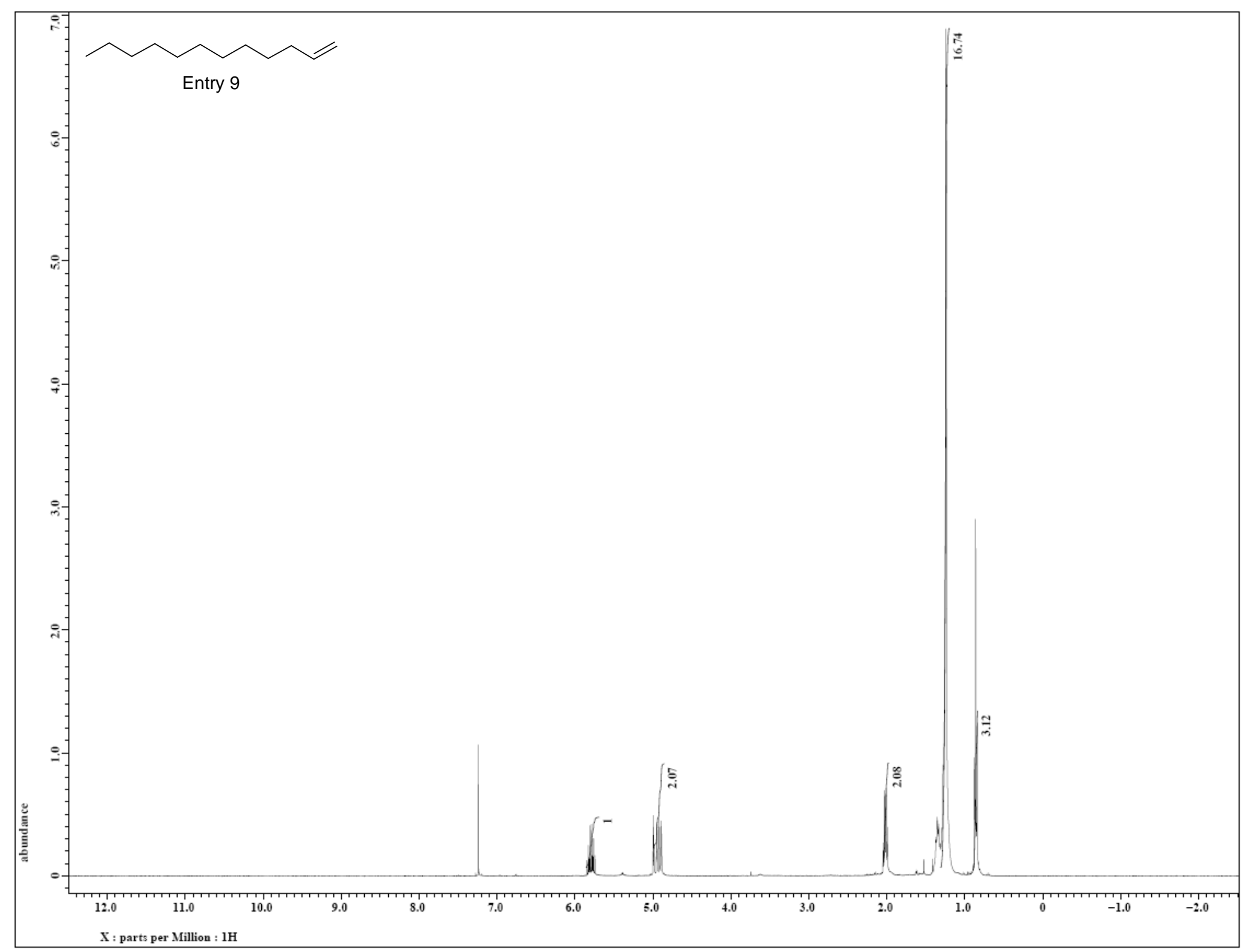




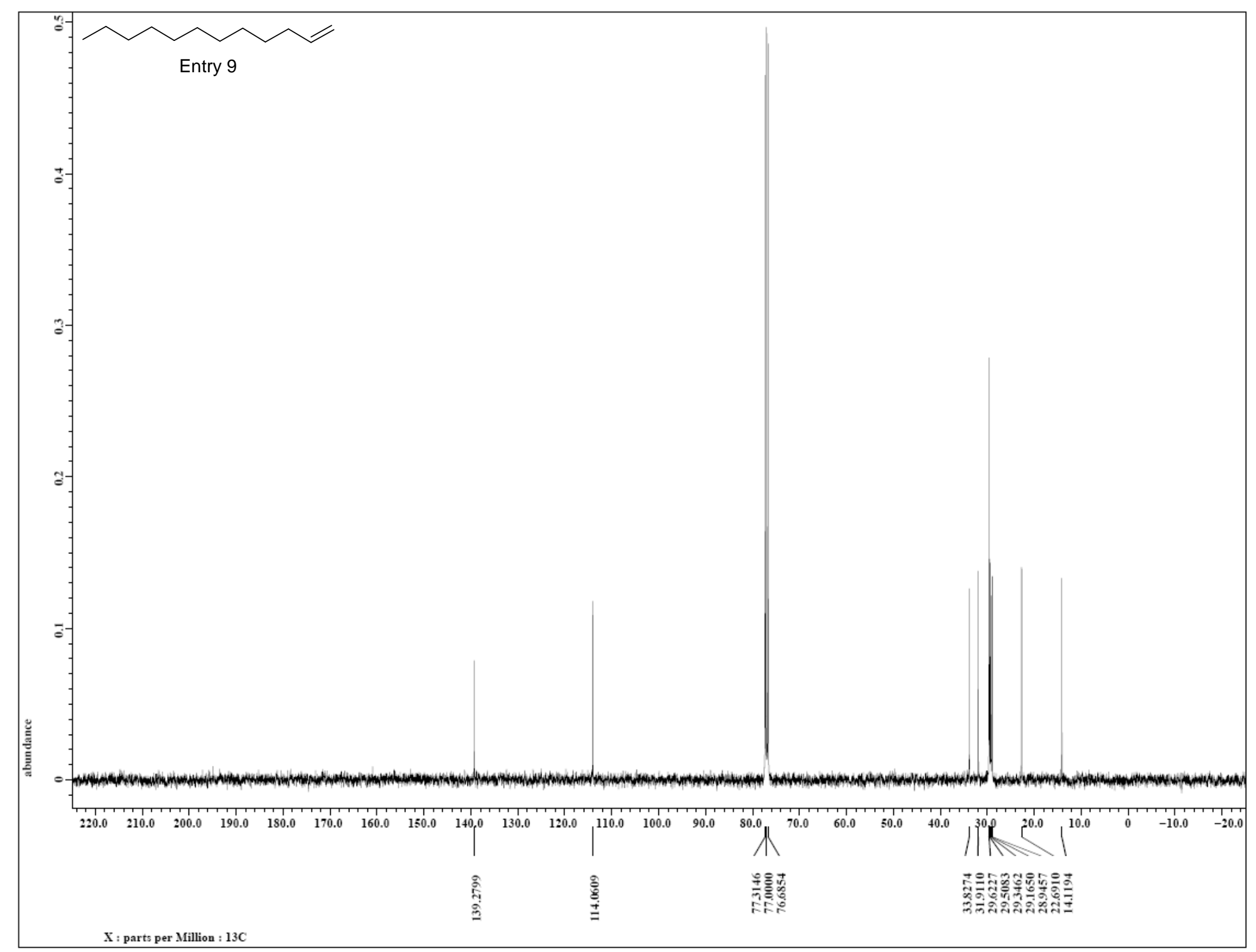



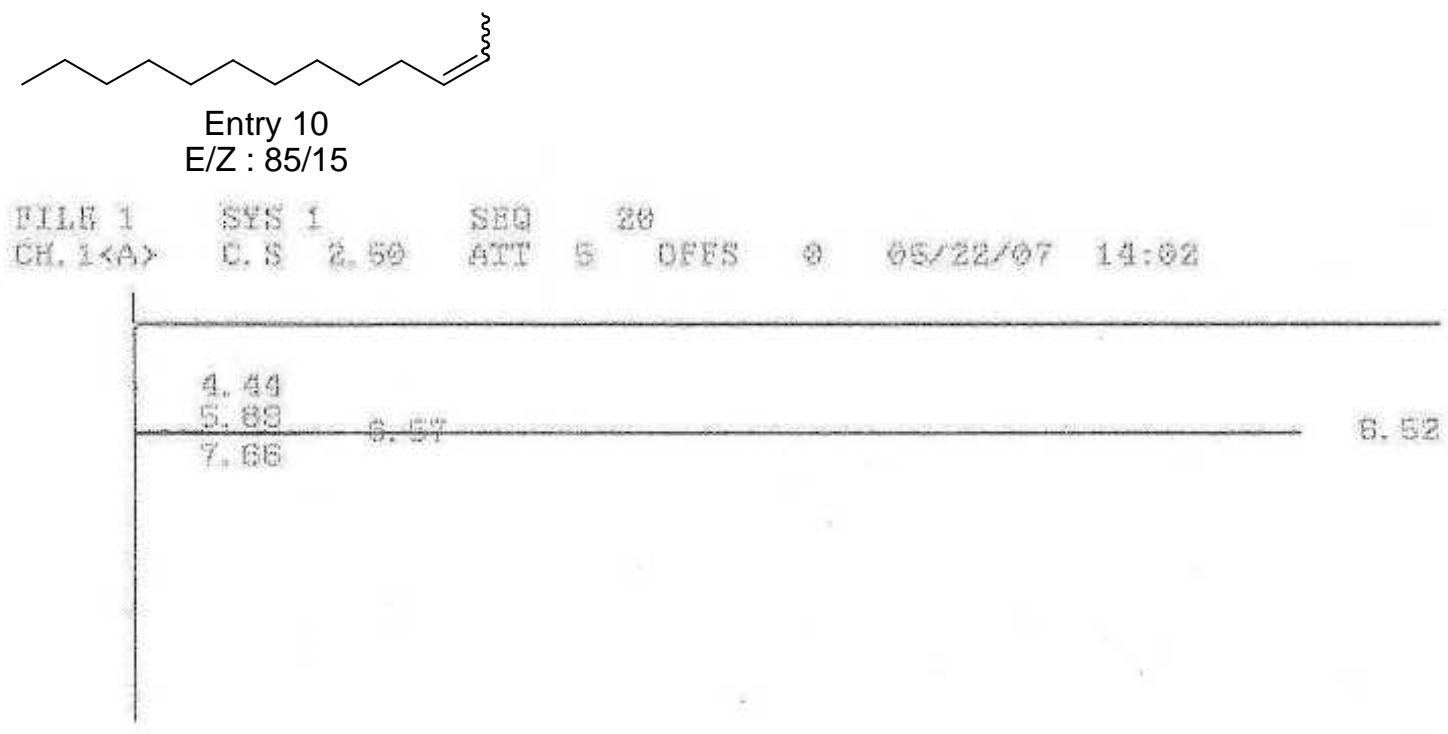

D-7598 IMU.

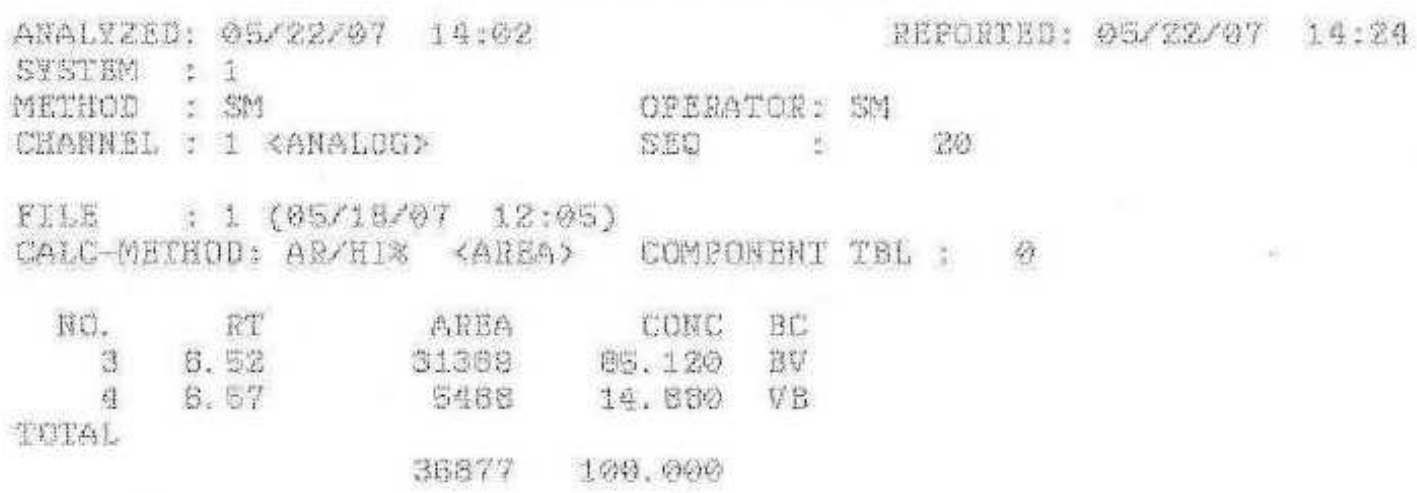




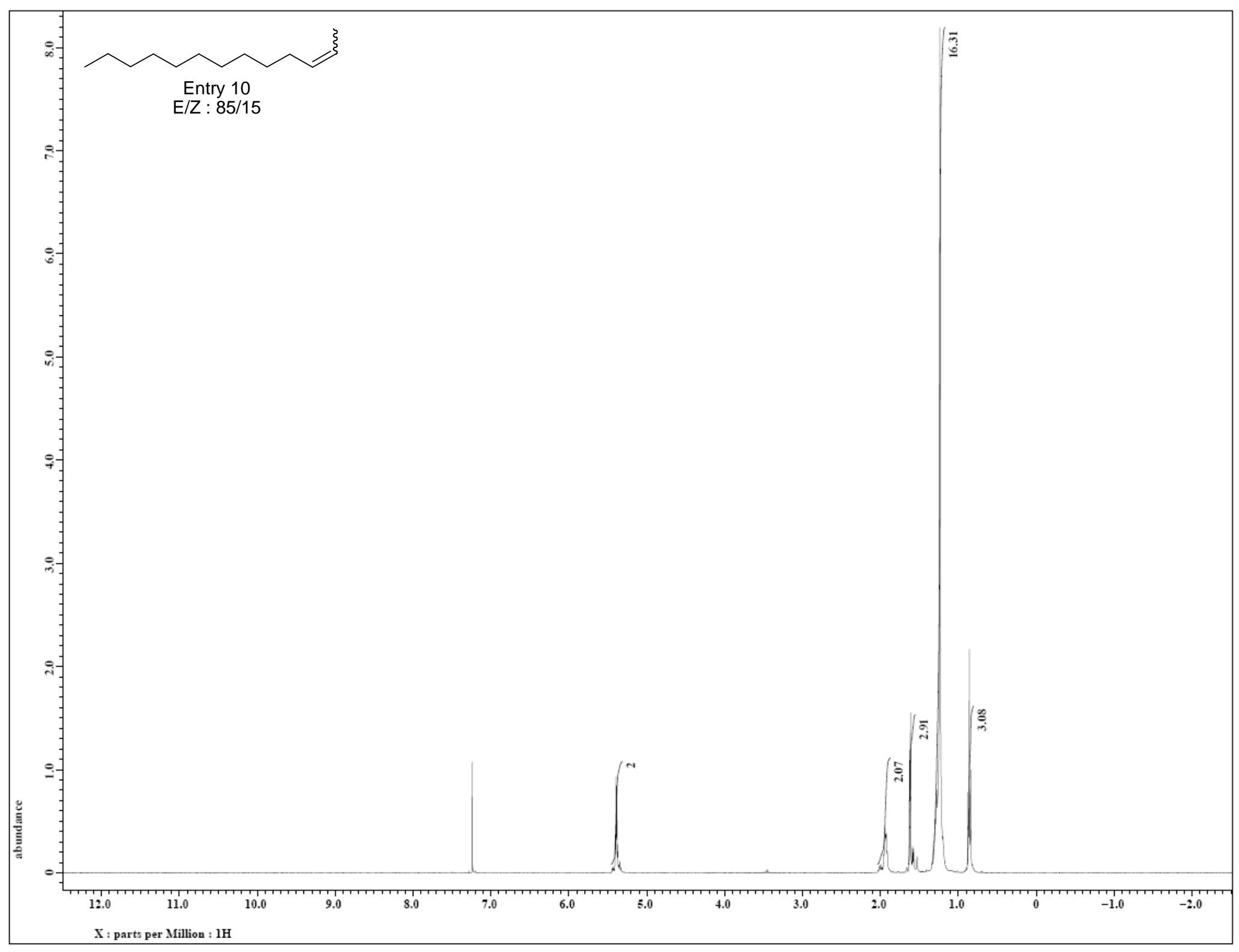




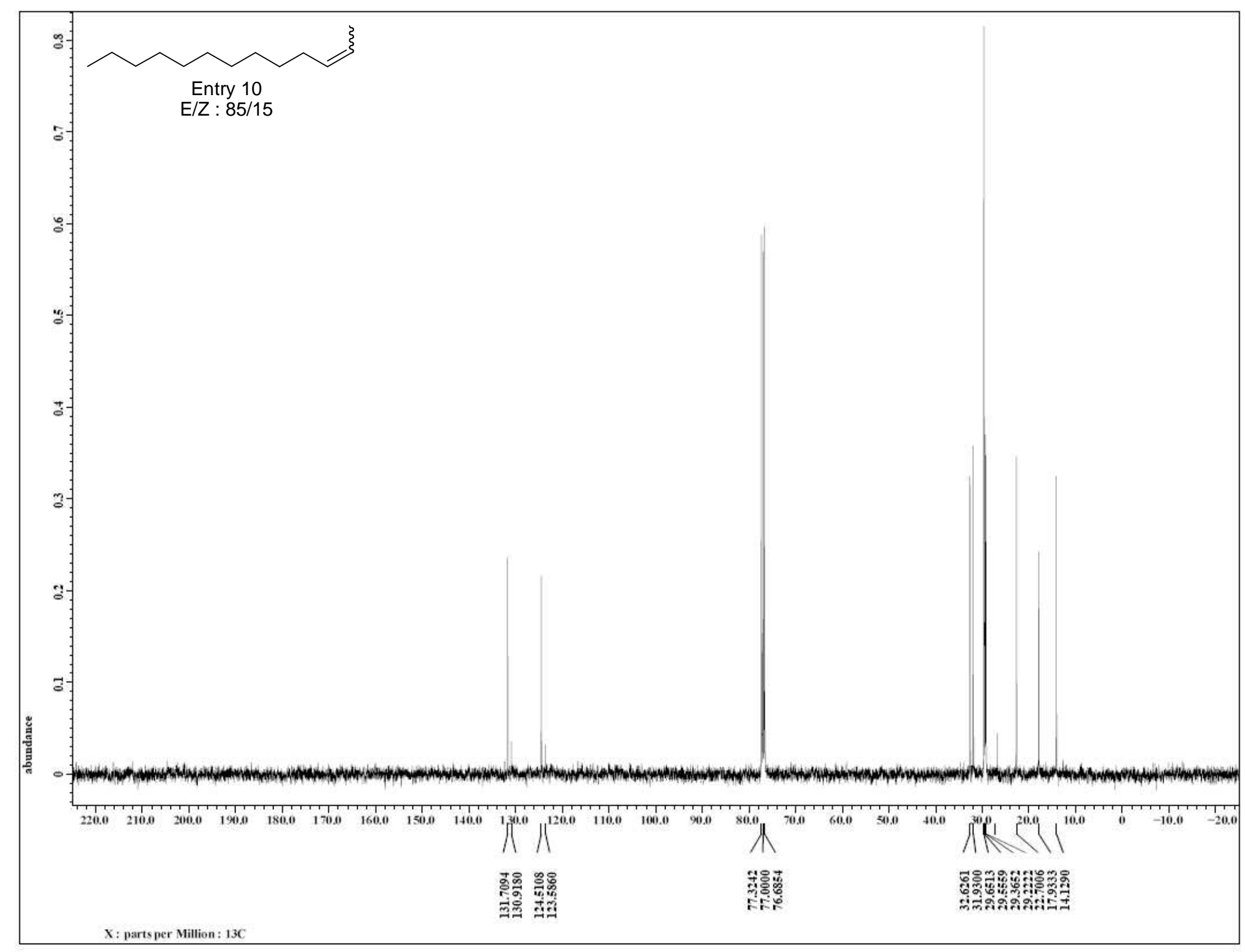




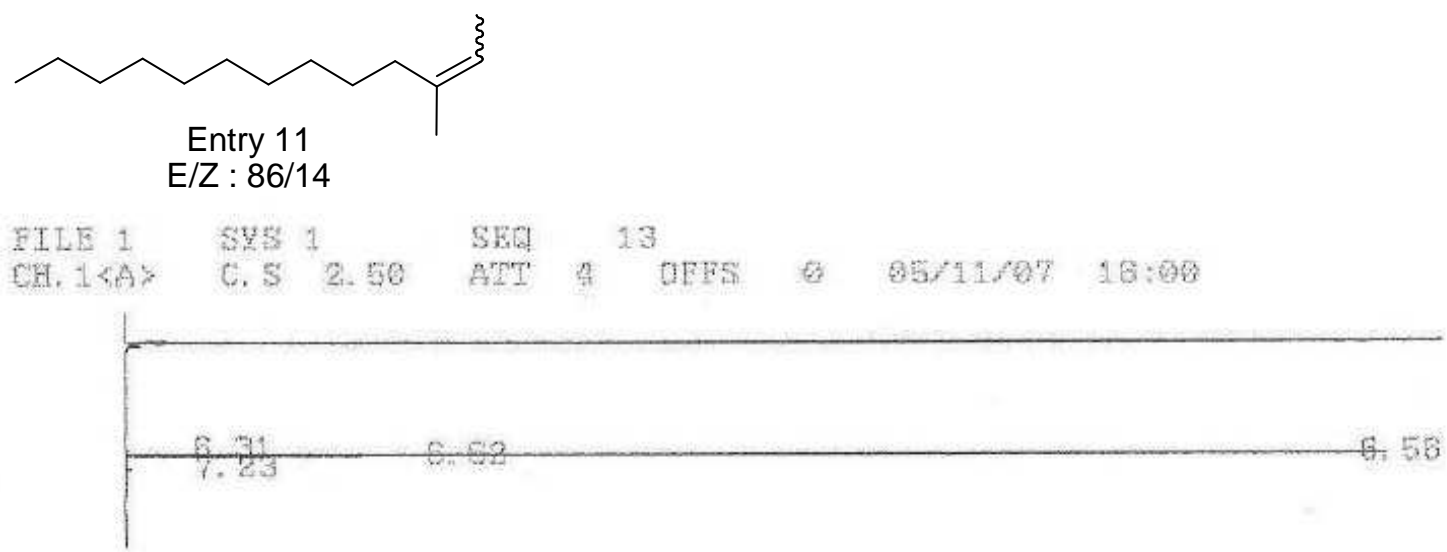

D-7500 HNTEGRATOP PEPDPT

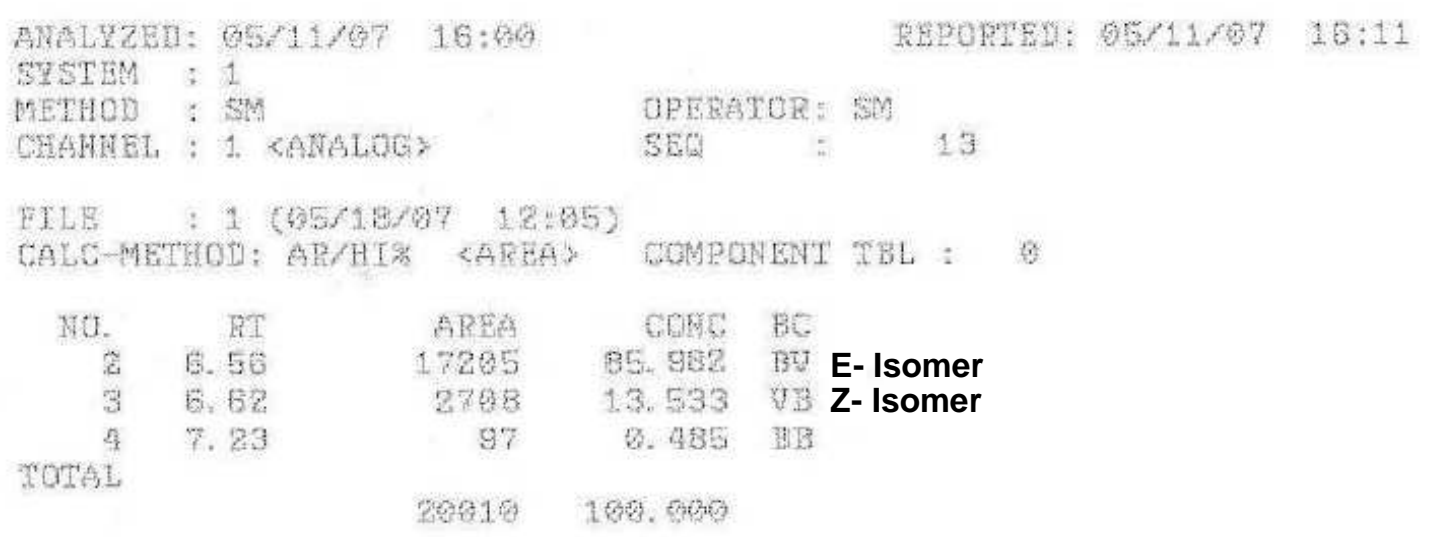




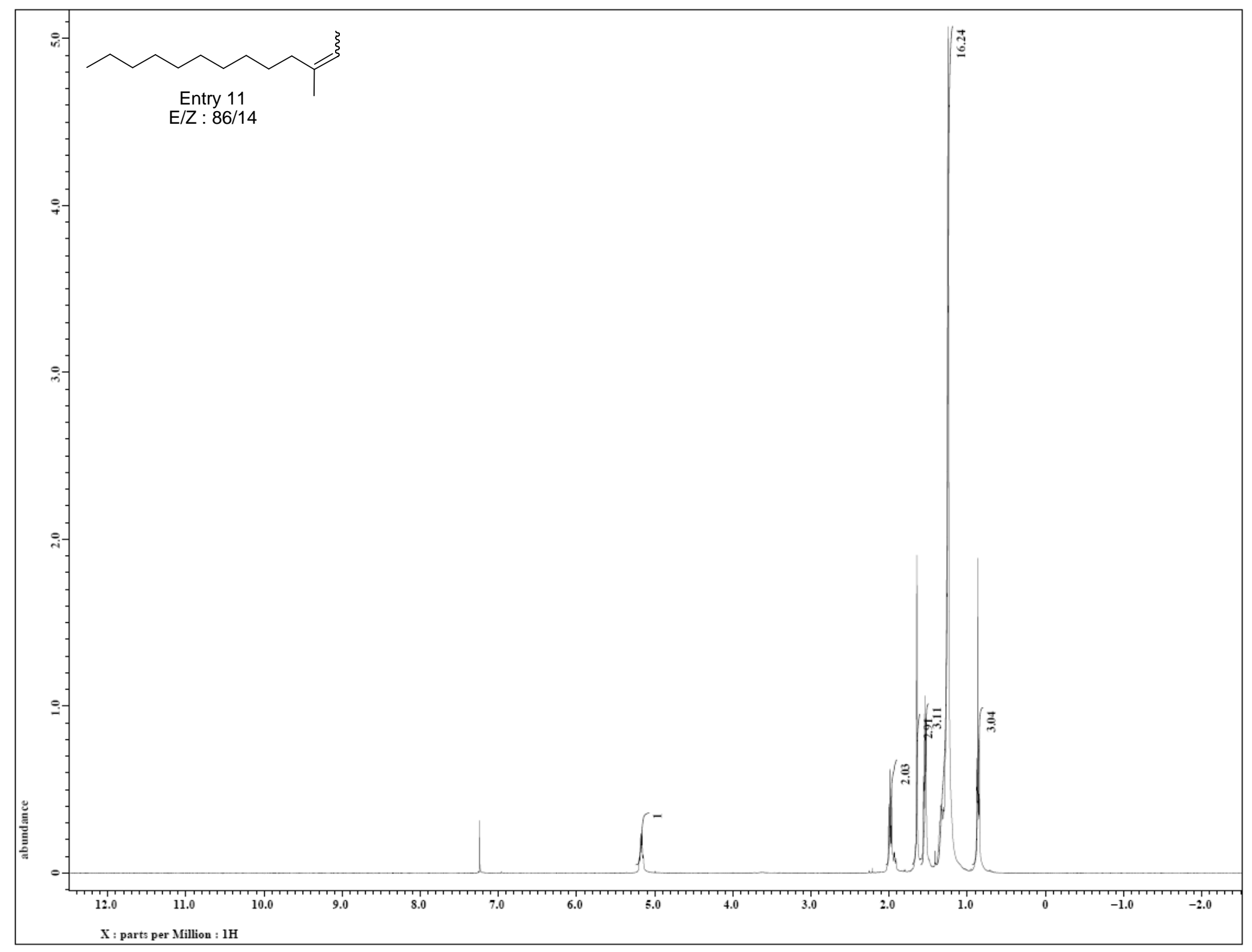




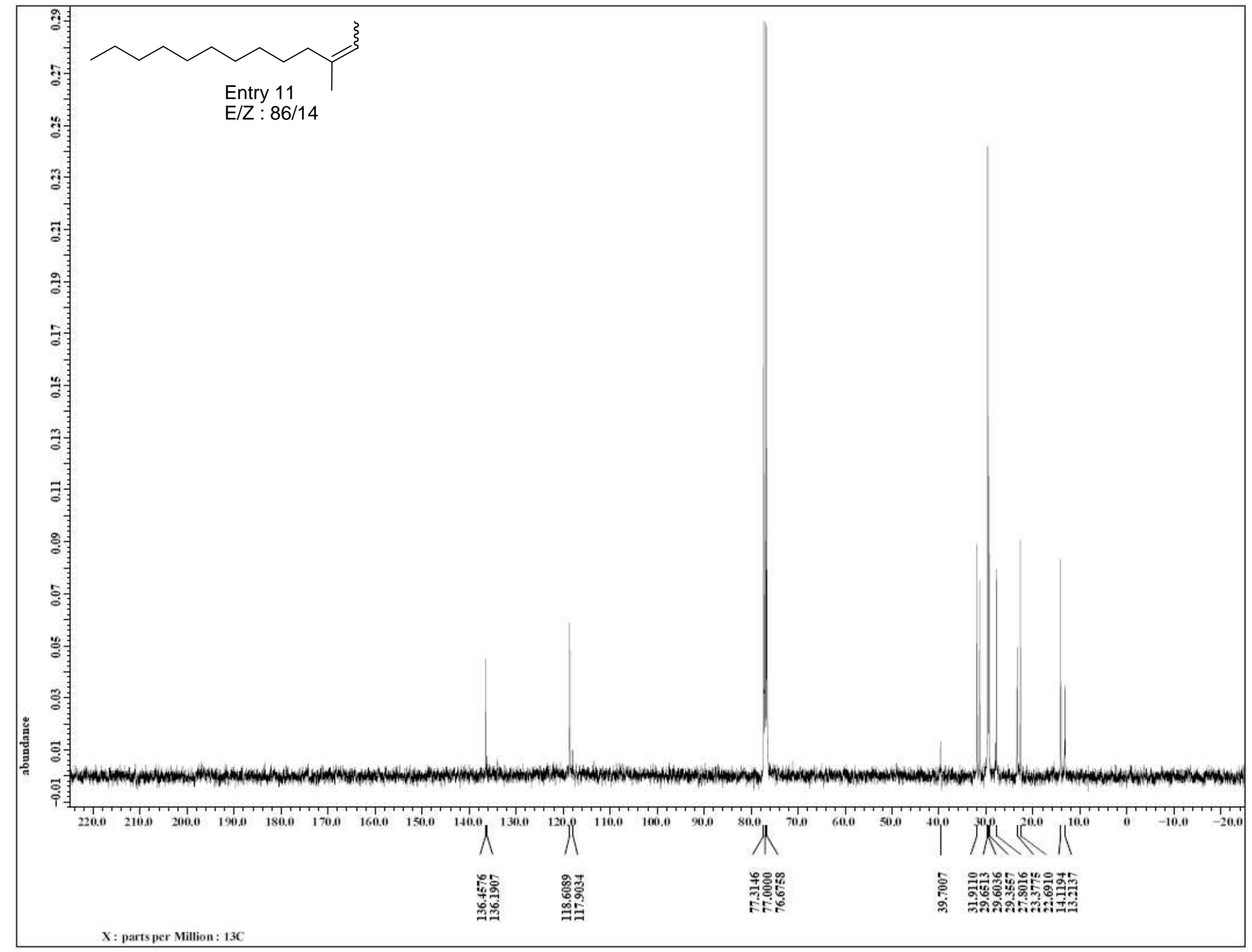




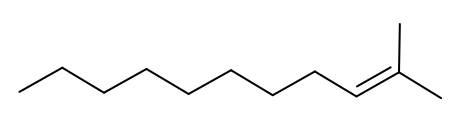

Entry 12

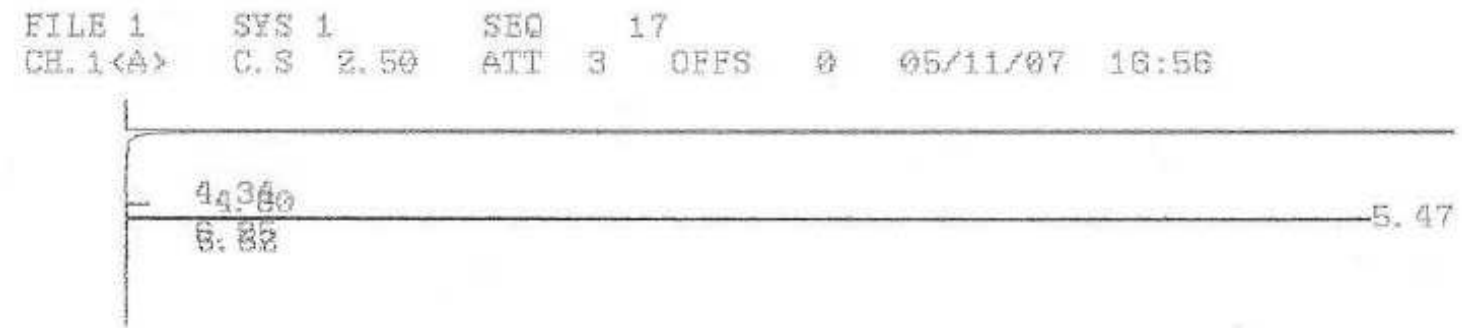

D-7500 INTRGRATOR RERORT

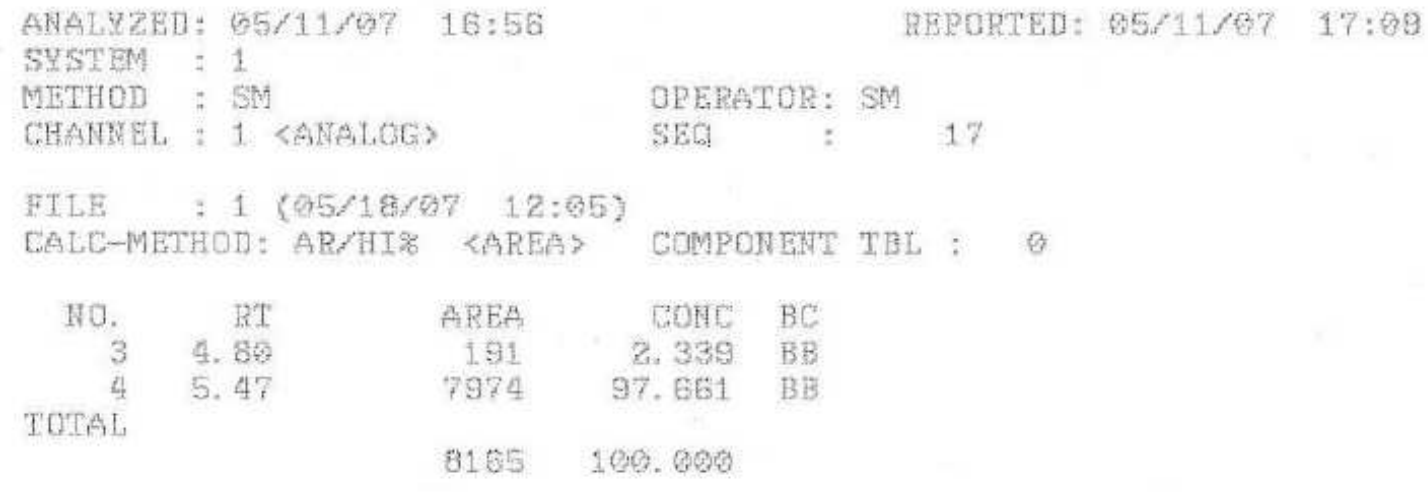




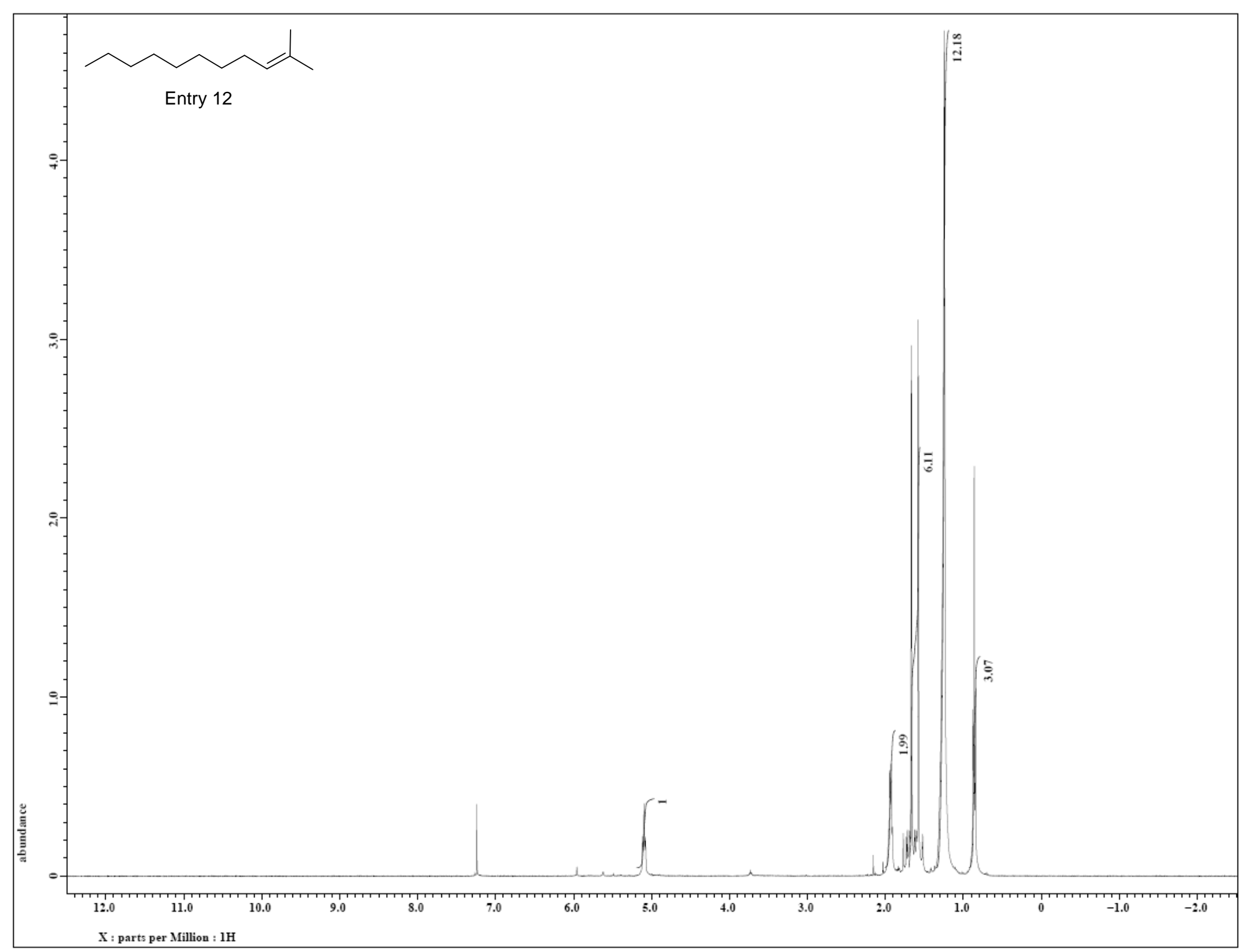

के 


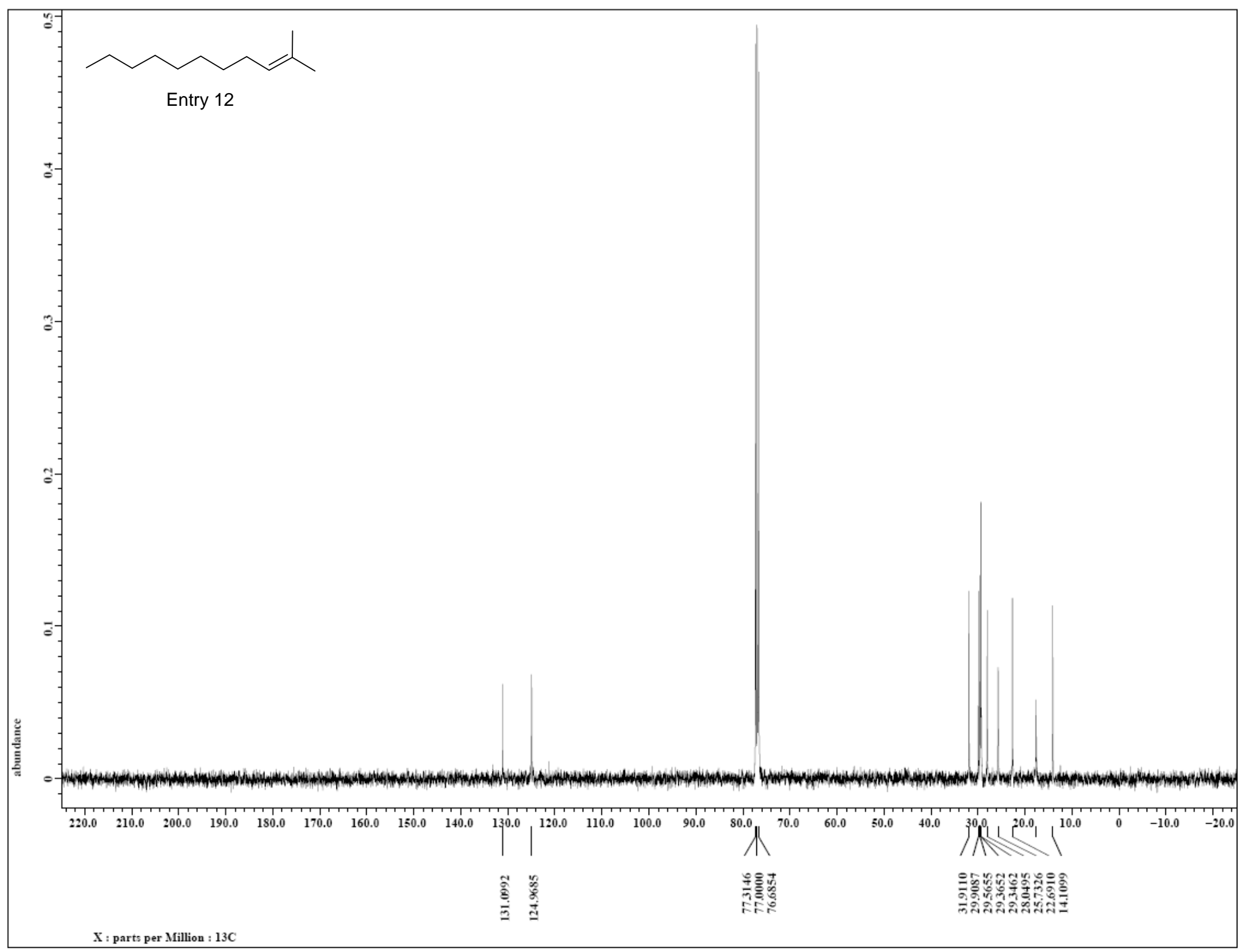

部 


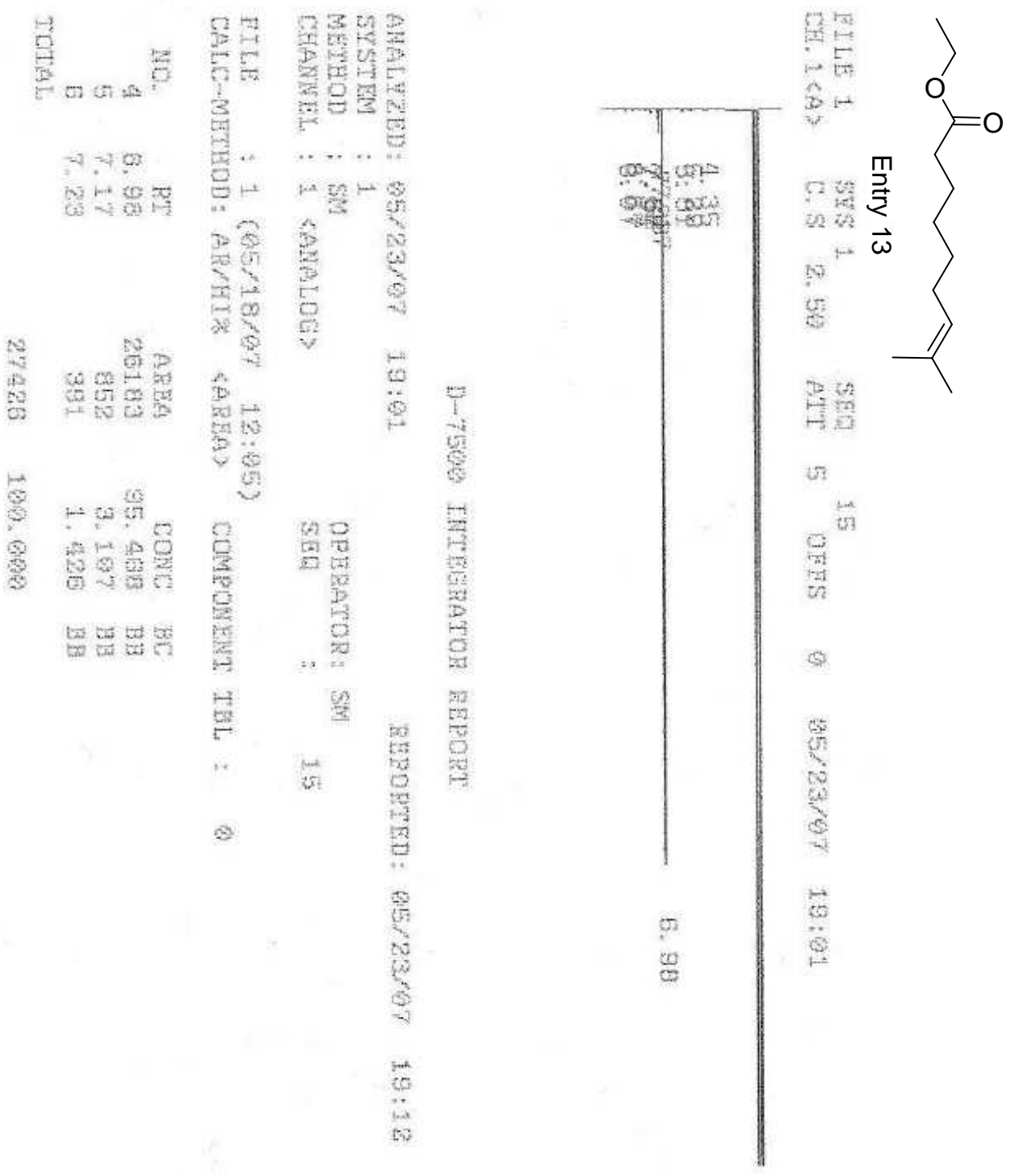




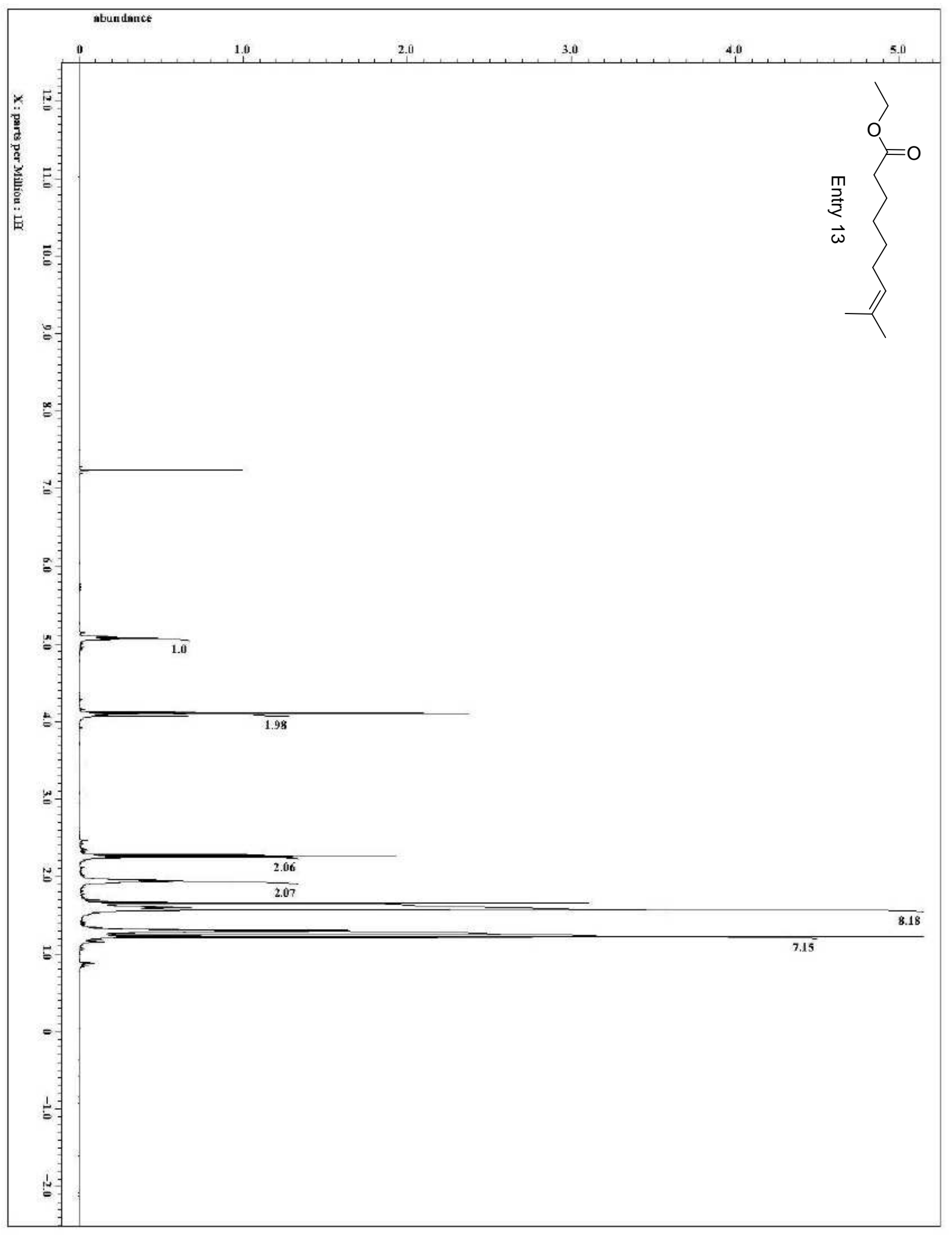




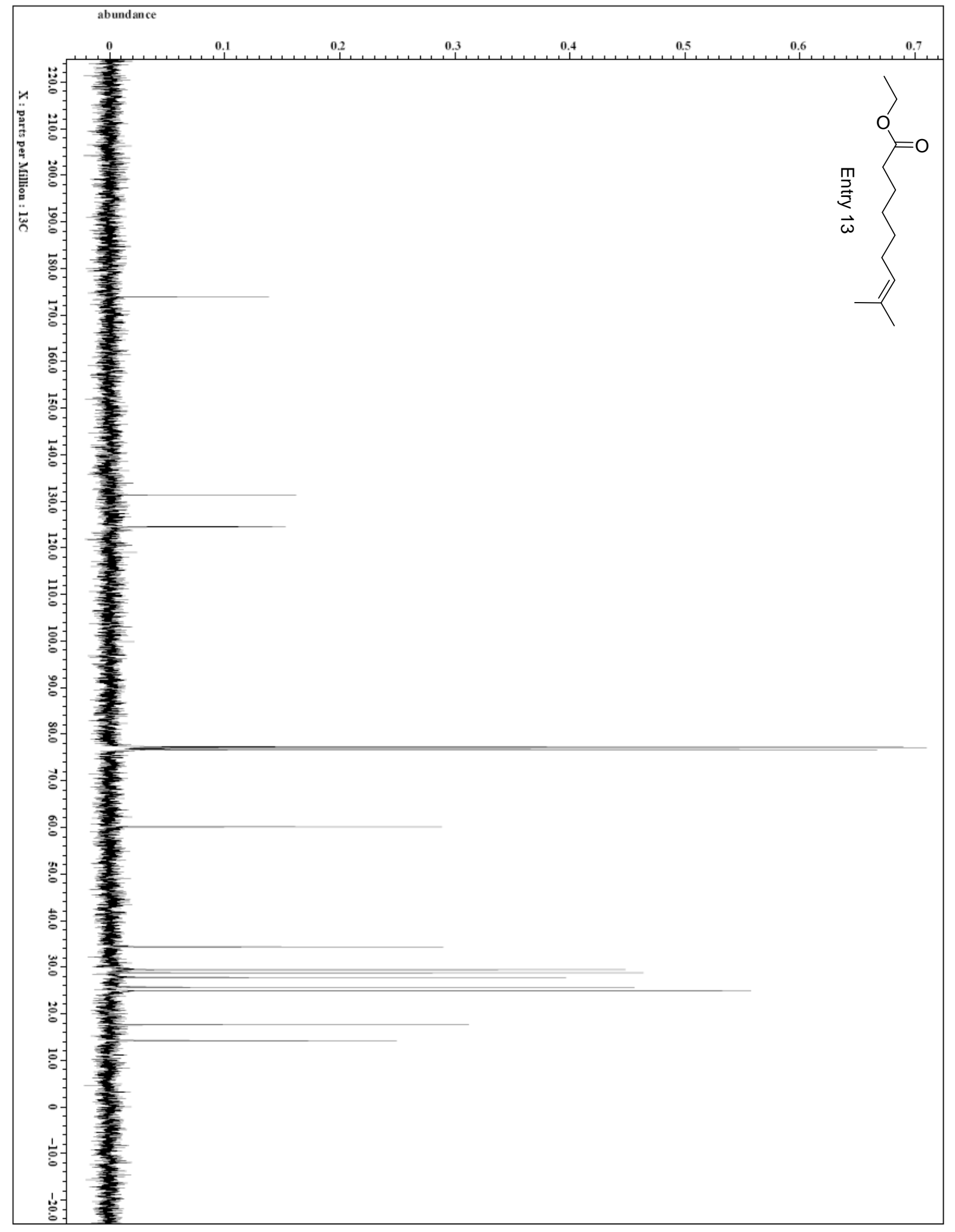




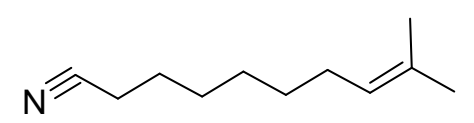

Entry 14

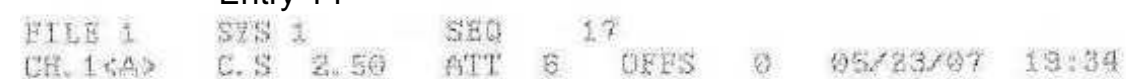

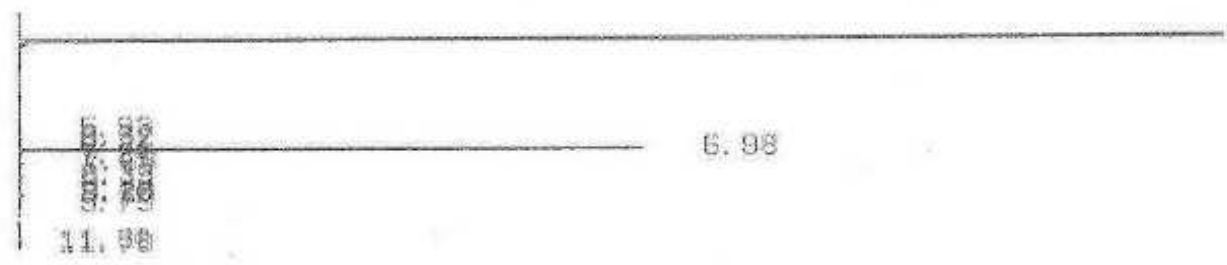

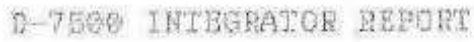

ALALEZED: \$5 $23 / 42$ 19:3 SUSTE⿱

METHOD : 5 M

GHAHNEL : 1 《AMALDIS

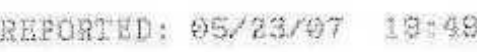

FILU : 1 (205618\%07 12:35)

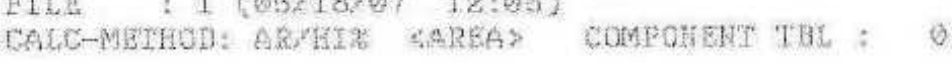

\begin{tabular}{|c|c|c|c|c|}
\hline Mo. & nI & $A \cap E A$ & Ealdo & \\
\hline z & 5,37 & 154 & 8.363 & \\
\hline 4 & E. 98 & 48814 & 57.437 & \\
\hline 7 & 7. 36 & 333 & 6. 445 & \\
\hline 9 & 83. 35 & 181. & 9. 361 & \\
\hline 9 & 8. 39 & 143 & 6.285 & \\
\hline i1 & 5. 200 & 137 & 0.272 & \\
\hline A.E & 9.88 & 215 & c. 929 & \\
\hline 14 & 21.60 & 168 & 0. 336 & \\
\hline 15 & 11.78 & 138 & Q. 276 & \\
\hline & & $5 \%$ & $163.60 b$ & \\
\hline
\end{tabular}




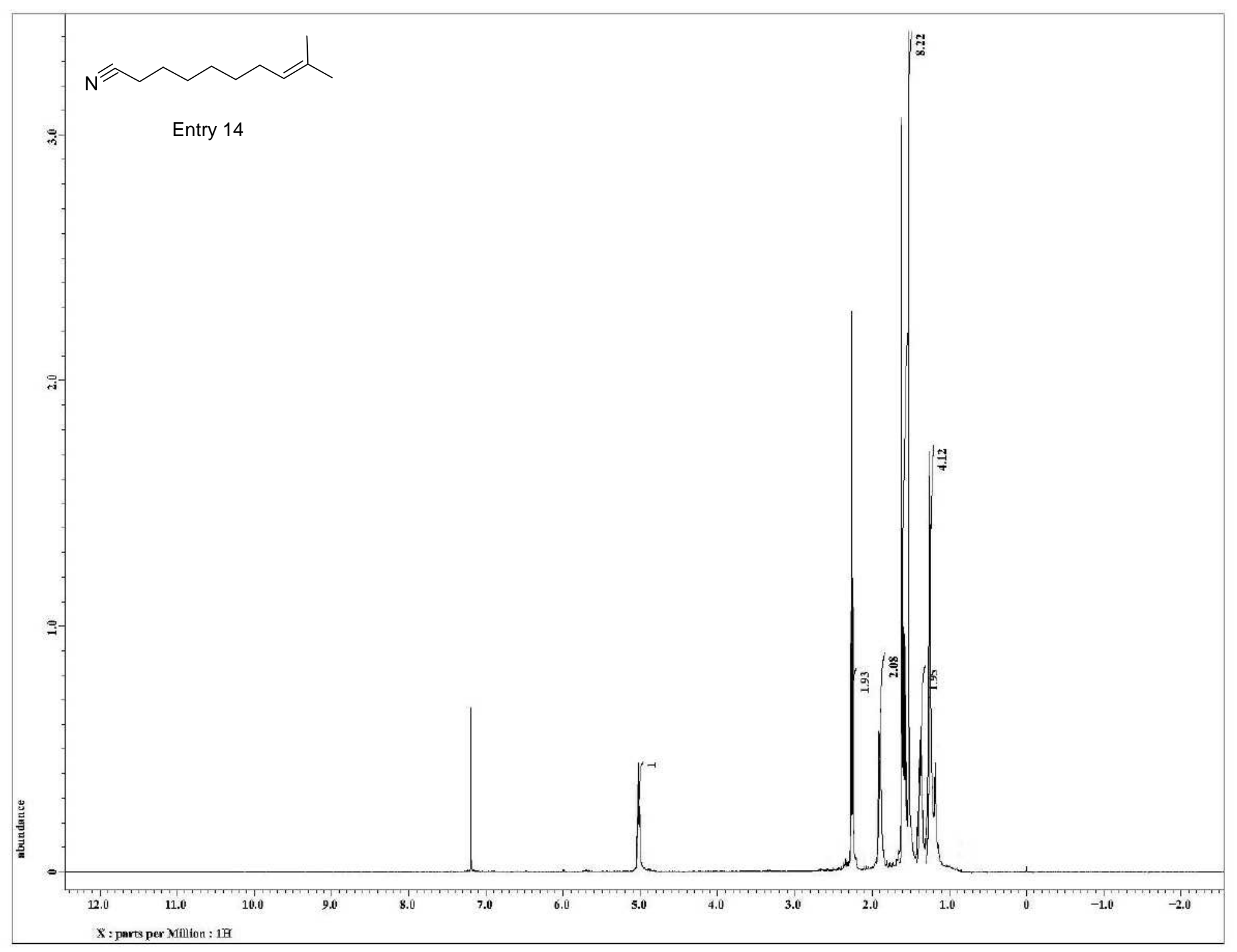




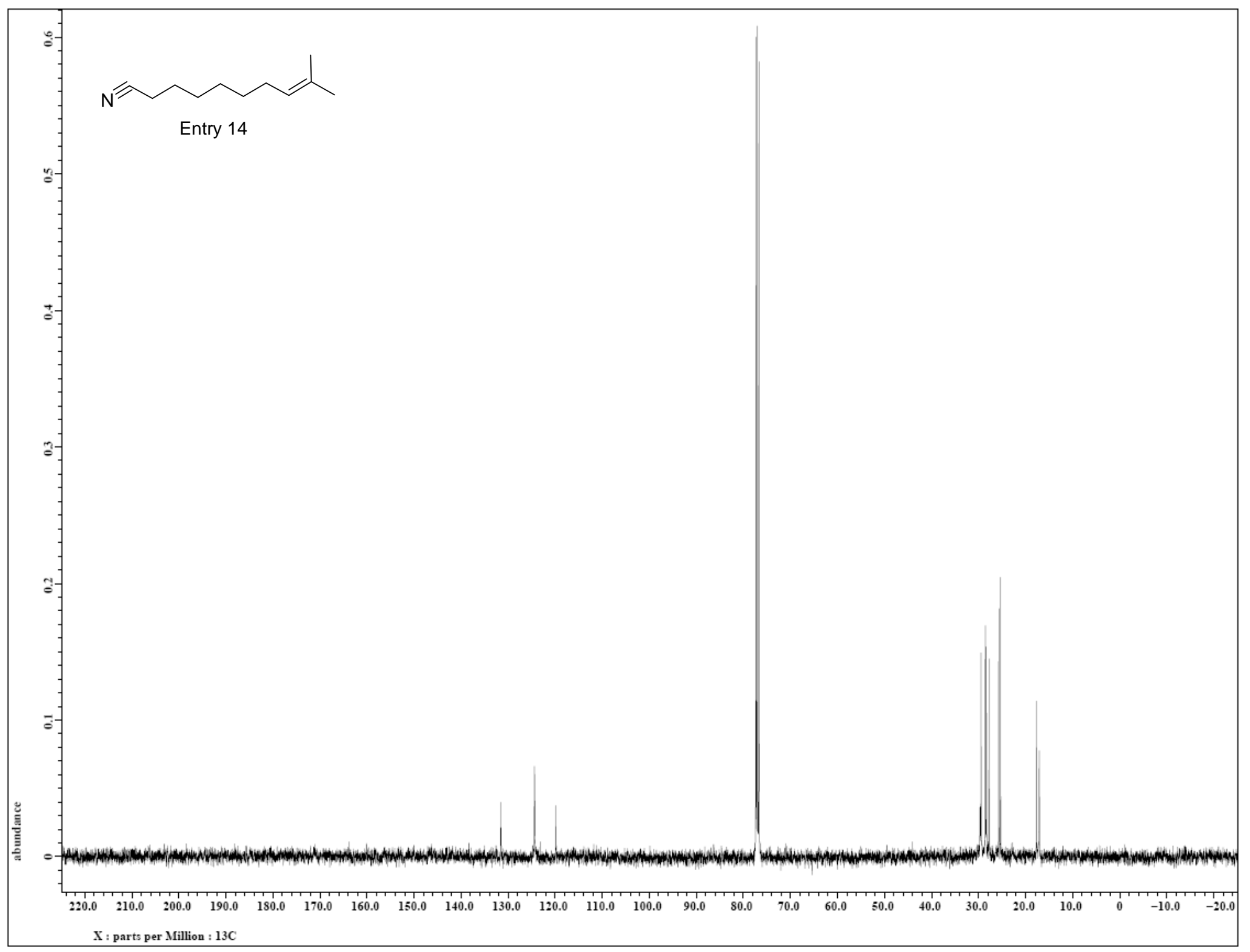




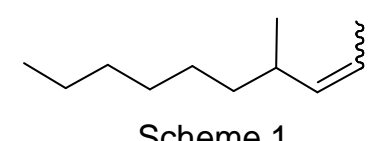

Scheme 1

Z/E : $97 / 3$

CH. I C.S 2.50 ATT 1 OFFS $\theta \quad 00 / 00 / 90 \quad 00: 02$

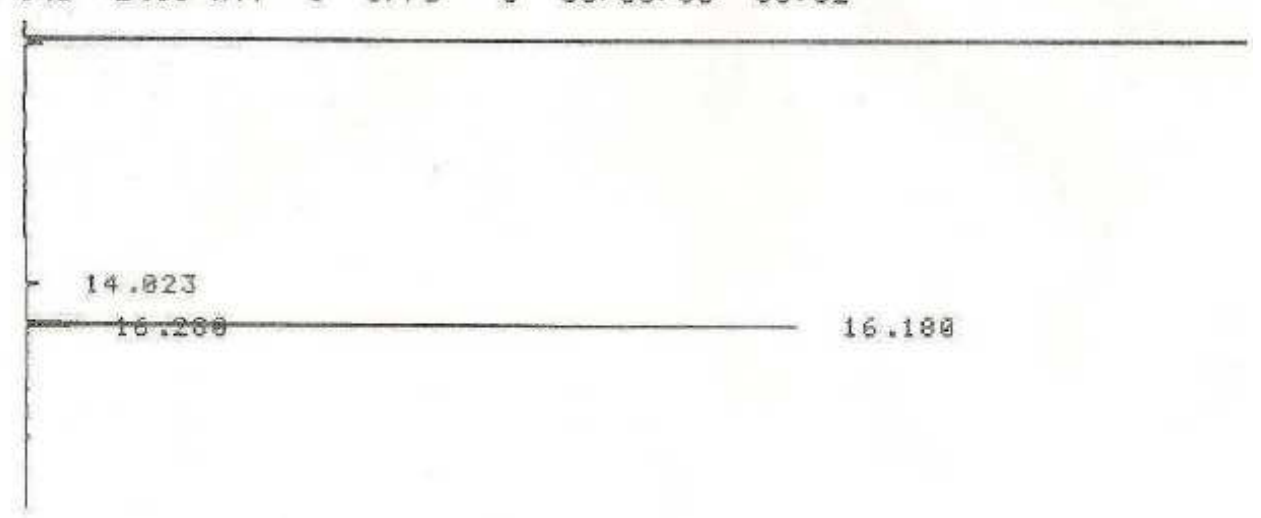

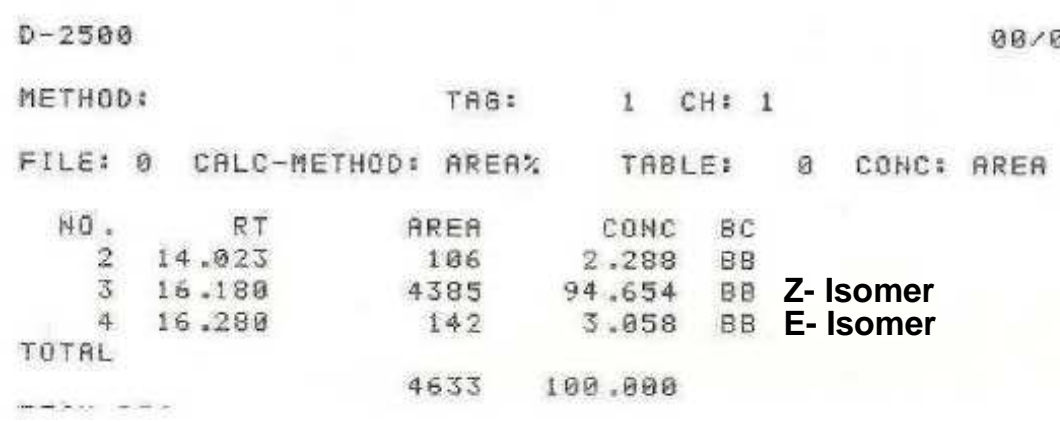

$3 / 89 / 80 \quad 80: 82$ 


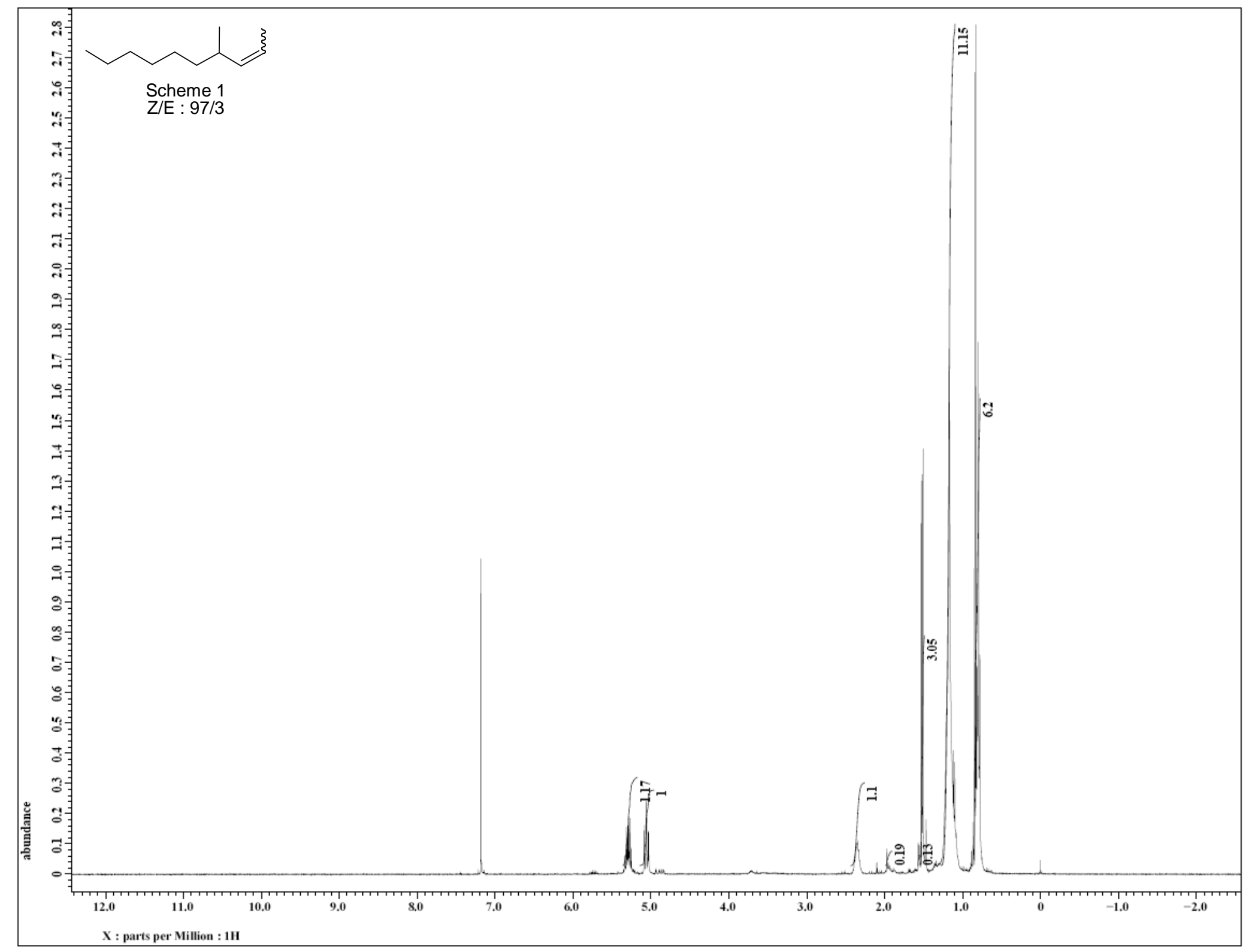




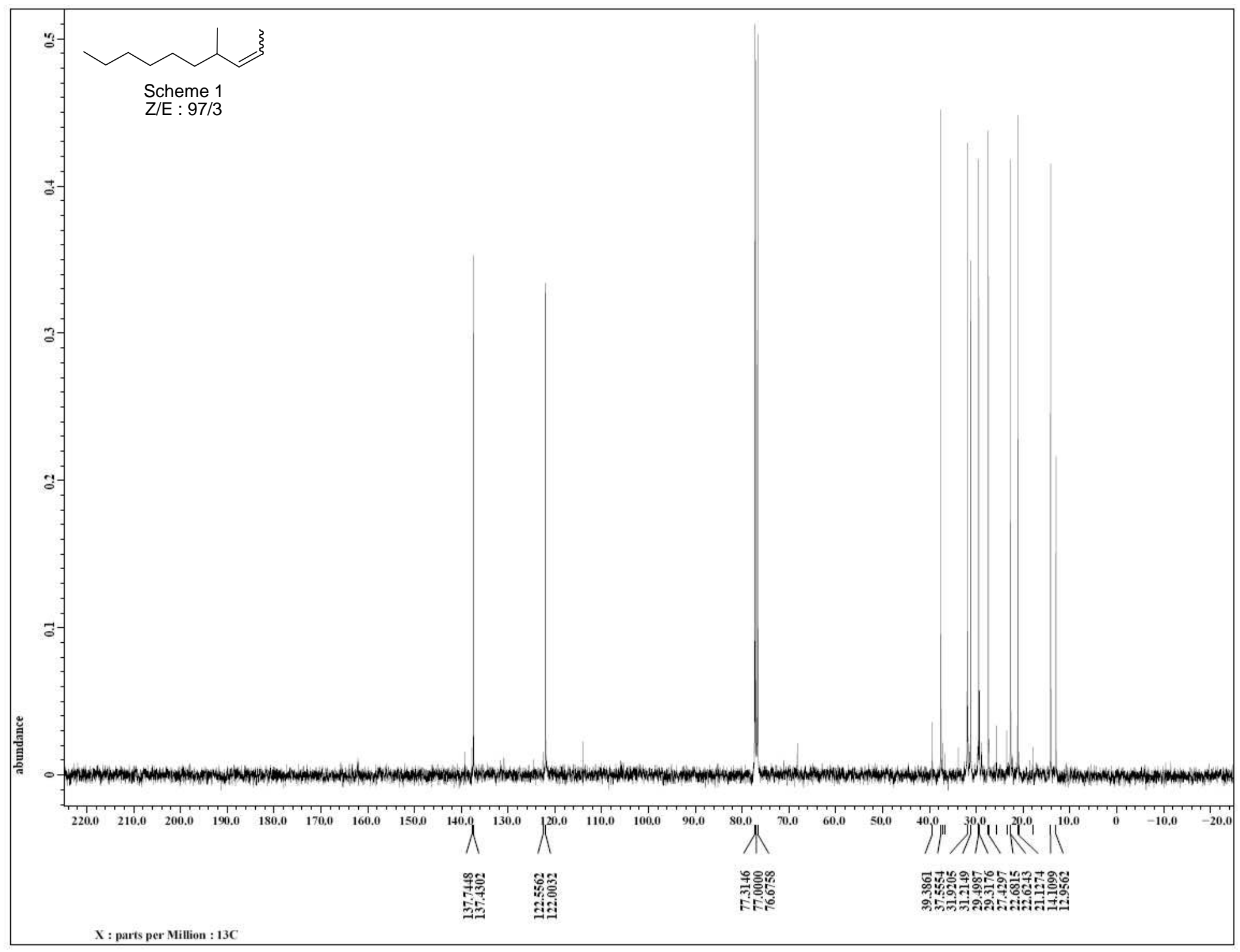

호 


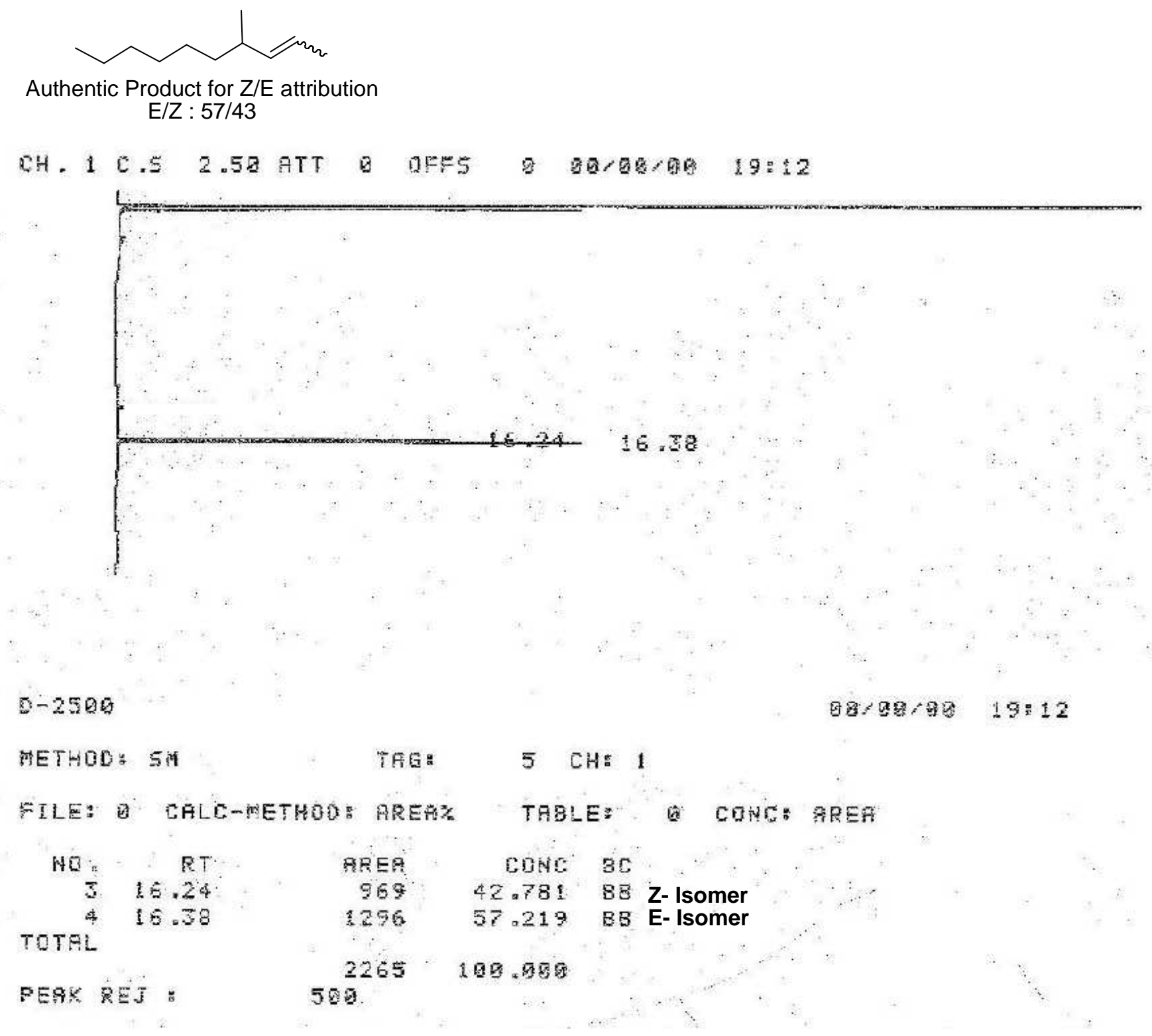




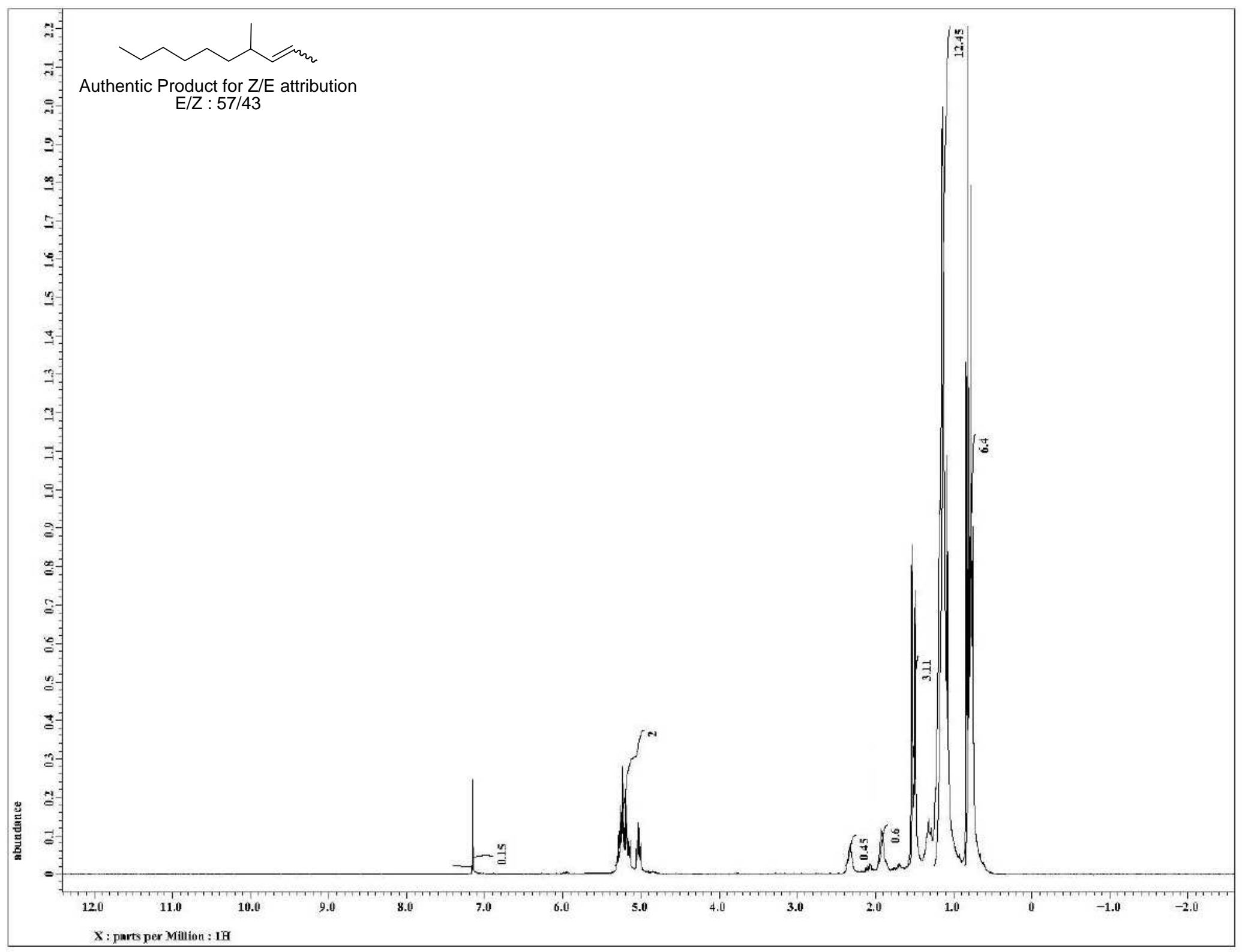




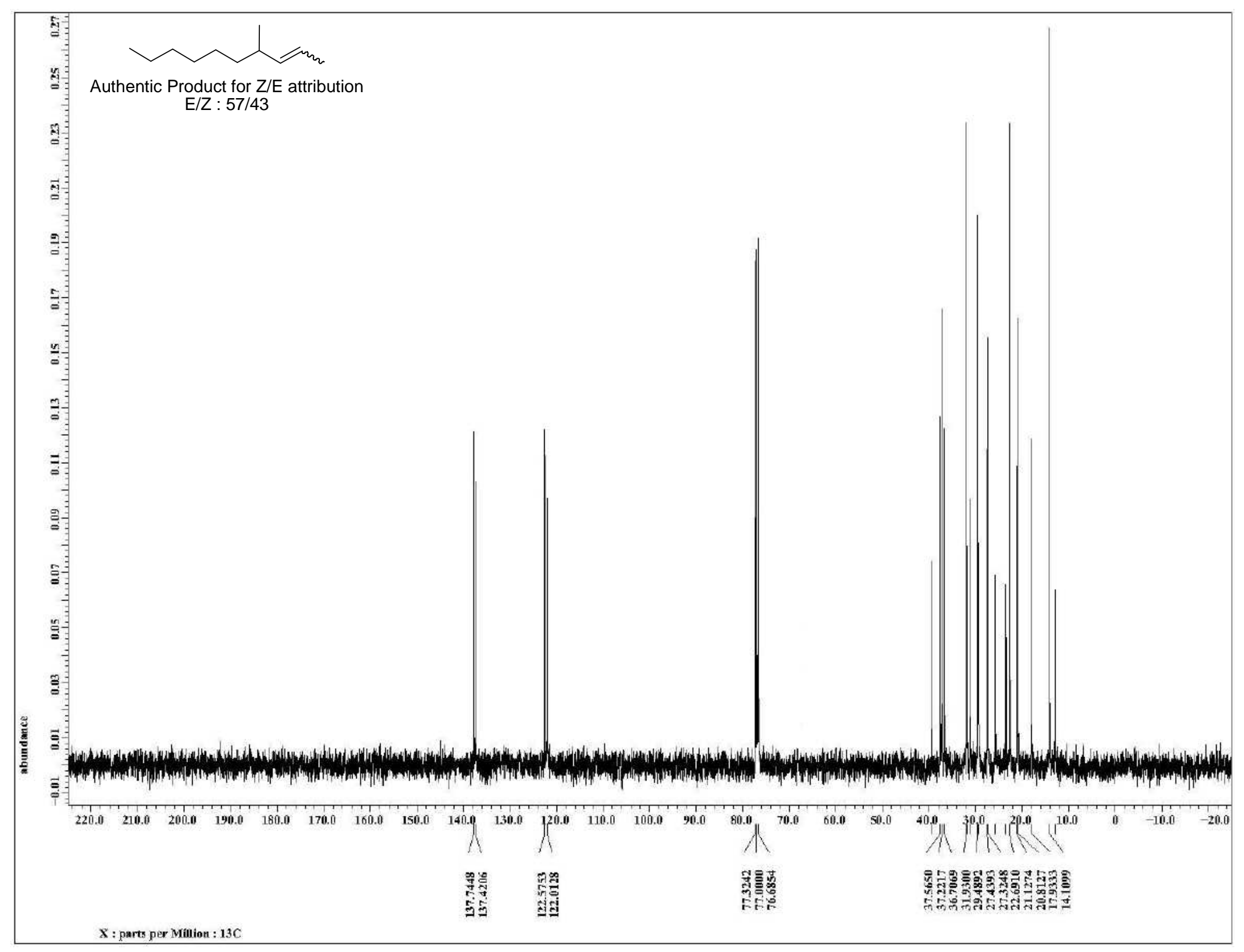

